\title{
Deterministic Parallel Fixpoint Computation
}

\author{
SUNG KOOK KIM, University of California, Davis, U.S.A. \\ ARNAUD J. VENET, Facebook, Inc., U.S.A. \\ ADITYA V. THAKUR, University of California, Davis, U.S.A.
}

\begin{abstract}
It reduces the problem of extracting properties of a program to computing an approximation of the least fixpoint of a system of equations. The de facto approach for computing this approximation uses a sequential algorithm based on weak topological order (WTO). This paper presents a deterministic parallel algorithm for fixpoint computation by introducing the notion of weak partial order (WPO). We present an algorithm for constructing a WPO in almost-linear time. Finally, we describe Pıкоs, our deterministic parallel abstract interpreter, which extends the sequential abstract interpreter IKOS. We evaluate the performance and scalability of PIKOS on a suite of $1017 \mathrm{C}$ programs. When using 4 cores, Pikos achieves an average speedup of 2.06x over IKOS, with a maximum speedup of 3.63x. When using 16 cores, PIKos achieves a maximum speedup of $10.97 x$.
\end{abstract}

CCS Concepts: • Software and its engineering $\rightarrow$ Automated static analysis; $\bullet$ Theory of computation $\rightarrow$ Program analysis.

Additional Key Words and Phrases: Abstract interpretation, Program analysis, Concurrency

ACM Reference Format:

Sung Kook Kim, Arnaud J. Venet, and Aditya V. Thakur. 2020. Deterministic Parallel Fixpoint Computation. Proc. ACM Program. Lang. 4, POPL, Article 14 (January 2020), 33 pages. https://doi.org/10.1145/3371082

\section{INTRODUCTION}

Program analysis is a widely adopted approach for automatically extracting properties of the dynamic behavior of programs [Balakrishnan et al. 2010; Ball et al. 2004; Brat and Venet 2005; Delmas and Souyris 2007; Jetley et al. 2008]. Program analyses are used, for instance, for program optimization, bug finding, and program verification. To be effective, a program analysis needs to be efficient, precise, and deterministic (the analysis always computes the same output for the same input program) [Bessey et al. 2010]. This paper aims to improve the efficiency of program analysis without sacrificing precision or determinism.

Abstract interpretation [Cousot and Cousot 1977] is a general framework for expressing static program analyses. A typical use of abstract interpretation to determine program invariants involves:

C1 An abstract domain $\mathcal{A}$ that captures relevant program properties. Abstract domains have been developed to perform, for instance, numerical analysis [Cousot and Halbwachs 1978; Miné 2004, 2006; Oulamara and Venet 2015; Singh et al. 2017; Venet 2012], heap analysis [Rinetzky et al. 2005; Wilhelm et al. 2000], and information flow [Giacobazzi and Mastroeni 2004].

Authors' addresses: Sung Kook Kim, Computer Science, University of California, Davis, Davis, California, 95616, U.S.A., sklkim@ucdavis.edu; Arnaud J. Venet, Facebook, Inc. Menlo Park, California, 94025, U.S.A., ajv@fb.com; Aditya V. Thakur, Computer Science, University of California, Davis, Davis, California, 95616, U.S.A., avthakur@ucdavis.edu.

This work is licensed under a Creative Commons Attribution 4.0 International License.

(c) 2020 Copyright held by the owner/author(s).

2475-1421/2020/1-ART14

https://doi.org/10.1145/3371082

Proc. ACM Program. Lang., Vol. 4, No. POPL, Article 14. Publication date: January 2020 
C2 An equation system $\mathcal{X}=F(\mathcal{X})$ over $\mathcal{A}$ that captures the abstract program behavior:

$$
X_{1}=F_{1}\left(X_{1}, \ldots, X_{n}\right), \quad X_{2}=F_{1}\left(X_{1}, \ldots, X_{n}\right), \quad \ldots, \quad X_{n}=F_{n}\left(X_{1}, \ldots, X_{n}\right)
$$

Each index $i \in[1, n]$ corresponds to a control point of the program, the unknowns $\mathcal{X}_{i}$ of the system correspond to the invariants to be computed for these control points, and each $F_{i}$ is a monotone operator incorporating the abstract transformers and control flow of the program.

C3 Computing an approximation of the least fixpoint of Eq. 1. The exact least solution of the system can be computed using Kleene iteration starting from the least element of $\mathcal{A}^{n}$ provided $\mathcal{A}$ is Noetherian. However, most interesting abstract domains require the use of widening to ensure termination, which may result in an over-approximation of the invariants of the program. A subsequent narrowing iteration tries to improve the post solution via a downward fixpoint iteration. In practice, abstract interpreters compute an approximation of the least fixpoint. In this paper, we use "fixpoint" to refer to such an approximation of the least fixpoint.

The iteration strategy specifies the order in which the equations in Eq. 1 are applied during fixpoint computation and where widening is performed. For a given abstraction, the efficiency, precision, and determinism of an abstract interpreter depends on the iteration strategy. The iteration strategy is determined by the dependencies between the individual equations in Eq. 1. If this dependency graph is acyclic, then the optimal iteration strategy is any topological order of the vertices in the graph. This is not true when the dependency graph contains cycles. Furthermore, each cycle in the dependency graph needs to be cut by at least one widening point.

Since its publication, Bourdoncle's algorithm [Bourdoncle 1993] has become the de facto approach for computing an efficient iteration strategy for abstract interpretation. Bourdoncle's algorithm determines the iteration strategy from a weak topological order (WTO) of the vertices in the dependency graph corresponding to the equation system. However, there are certain disadvantages to Bourdoncle's algorithm: (i) the iteration strategy computed by Bourdoncle's algorithm is inherently sequential: WTO gives a total order of the vertices in the dependency graph; (ii) computing WTO using Bourdoncle's algorithm has a worst-case cubic time complexity; (iii) the mutually-recursive nature of Bourdoncle's algorithm makes it difficult to understand (even for seasoned practitioners of abstract interpretation); and (iv) applying Bourdoncle's algorithm, as is, to deep dependency graphs can result in a stack overflow in practice. ${ }^{1,2}$

This paper addresses the above disadvantages of Bourdoncle's algorithm by presenting a concurrent iteration strategy for fixpoint computation in an abstract interpreter (§5). This concurrent fixpoint computation can be efficiently executed on modern multi-core hardware. The algorithm for computing our iteration strategy has a worst-case almost-linear time complexity, and lends itself to a simple iterative implementation (§6). The resulting parallel abstract interpreter, however, remains deterministic: for the same program, all possible executions of the parallel fixpoint computation give the same result. In fact, the fixpoint computed by our parallel algorithm is the same as that computed by Bourdoncle's sequential algorithm $(\S 7)$.

To determine the concurrent iteration strategy, this paper introduces the notion of a weak partial $\operatorname{order}(W P O)$ for the dependency graph of the equation system $(\S 4)$. WPO generalizes the notion of WTO: a WTO is a linear extension of a WPO (§7). Consequently, the almost-linear time algorithm for WPO can also be used to compute WTO. The algorithm for WPO construction handles dependency graphs that are irreducible [Hecht and Ullman 1972; Tarjan 1973]. The key insight behind our approach is to adapt algorithms for computing loop nesting forests [Ramalingam 1999, 2002] to the problem of computing a concurrent iteration strategy for abstract interpretation.

\footnotetext{
${ }^{1}$ Workaround that prevents stack overflows in WTO computation https://github.com/facebook/redex/commit/6bbf8a5ddb.

${ }^{2}$ Possible stack overflow while computing WTO of a large CFG https://github.com/seahorn/crab/issues/18.
} 
We have implemented our concurrent fixpoint iteration strategy in a tool called Pikos (§8). Using a suite of $1017 \mathrm{C}$ programs, we compare the performance of PIKos against the state-of-the-art abstract interpreter IKOS [Brat et al. 2014], which uses Bourdoncle's algorithm (§9). When using 4 cores, PIKos achieves an average speedup of 2.06x over IKOS, with a maximum speedup of 3.63x We see that Pikos exhibits a larger speedup when analyzing programs that took longer to analyze using IKOS. PIKOs achieved an average speedup of $1.73 \mathrm{x}$ on programs for which IKOS took less than 16 seconds, while PIKOS achieved an average speedup of $2.38 \mathrm{x}$ on programs for which IKOS took greater than 508 seconds. The scalability of Pikos depends on the structure of the program being analyzed. When using 16 cores, Pikos achieves a maximum speedup of 10.97x.

The contributions of the paper are as follows:

- We introduce the notion of a weak partial order (WPO) for a directed graph (§4), and show how this generalizes the existing notion of weak topological order (WTO) (§7).

- We present a concurrent algorithm for computing the fixpoint of a set of equations $(\S 5)$.

- We present an almost-linear time algorithm for WPO and WTO construction (§6).

- We describe our deterministic parallel abstract interpreter Pıкоs (§8), and evaluate its performance on a suite of $\mathrm{C}$ programs $(\S 9)$.

$\S 2$ presents an overview of the technique; §3 presents mathematical preliminaries; $§ 10$ describes related work; §11 concludes.

\section{OVERVIEW}

Abstract interpretation is a general framework that captures most existing approaches for static program analyses and reduces extracting properties of programs to approximating their semantics [Cousot and Cousot 1977; Cousot et al. 2019]. Consequently, this section is not meant to capture all possible approaches to implementing abstract interpretation or describe all the complex optimizations involved in a modern implementation of an abstract interpreter. Instead it is only meant to set the appropriate context for the rest of the paper, and to capture the relevant high-level structure of abstract-interpretation implementations such as IKOS [Brat et al. 2014].

Fixpoint equations. Consider the simple program $P$ represented by its control flow graph (CFG) in Figure 1(a). We will illustrate how an abstract interpreter would compute the set of values that variable $x$ might contain at each program point $i$ in $P$. In this example, we will use the standard integer interval domain [Cousot and Cousot 1976, 1977] represented by the complete lattice $\langle$ Int $, \sqsubseteq, \perp, \top, \sqcup, \sqcap\rangle$ with $\operatorname{Int} \stackrel{\text { def }}{=}\{\perp\} \cup\{[l, u] \mid l, u \in \mathbb{Z} \wedge l \leq u\} \cup\{[-\infty, u] \mid u \in \mathbb{Z}\} \cup\{[l, \infty] \mid$ $l \in \mathbb{Z}\} \cup\{[-\infty, \infty]\}$. The partial order $\sqsubseteq$ on Int is interval inclusion with the empty interval $\perp=\emptyset$ encoded as $[\infty,-\infty]$ and $T=[-\infty, \infty]$.

Figure 1(b) shows the corresponding equation system $X=F(\mathcal{X})$, where $\mathcal{X}=\left(\mathcal{X}_{0}, \mathcal{X}_{1}, \ldots, \mathcal{X}_{8}\right)$. Each equation in this equation system is of the form $\mathcal{X}_{i}=F_{i}\left(\mathcal{X}_{0}, \mathcal{X}_{1}, \ldots, \mathcal{X}_{8}\right)$, where the variable $\mathcal{X}_{i} \in$ Int represents the interval value at program point $i$ in $\mathrm{P}$ and $F_{i}$ is monotone. The operator + represents the (standard) addition operator over Int. As is common (but not necessary), the dependencies among the equations reflect the CFG of program $P$.

The exact least solution $\mathcal{X}^{\text {lfp }}$ of the equation system $\mathcal{X}=F(\mathcal{X})$ would give the required set of values for variable $\mathrm{x}$ at program point $i$. Let $\mathcal{X}^{0}=(\perp, \perp, \ldots, \perp)$ and $\mathcal{X}^{i+1}=F\left(\mathcal{X}^{i}\right), i \geq 0$ represent the standard Kleene iterates, which converge to $\mathcal{X}^{\mathrm{lfp}}$.

Chaotic iteration. Instead of applying the function $F$ during Kleene iteration, one can use chaotic iterations [Cousot 1977; Cousot and Cousot 1977] and apply the individual equations $F_{i}$. The order in which the individual equations are applied is determined by the chaotic iteration strategy. 


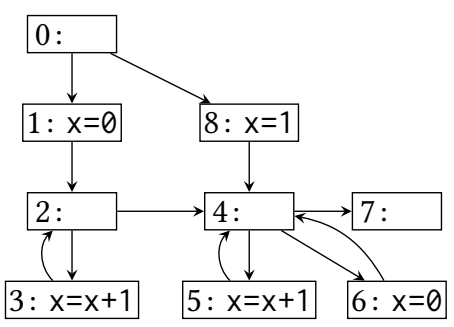

(a)

$X_{0}=\top$
$X_{1}=[0,0]$
$X_{2}=X_{1} \sqcup X_{3}$
$X_{3}=X_{2}+[1,1]$
$X_{4}=X_{2} \sqcup X_{8} \sqcup X_{5} \sqcup X_{6}$
$X_{5}=X_{4}+[1,1]$
$X_{6}=[0,0]$
$X_{7}=X_{4}$
$X_{8}=[1,1]$

(b)

$$
\begin{aligned}
& X_{0}=\top \\
& X_{1}=[0,0] \\
& X_{2}=X_{2} \nabla\left(X_{1} \sqcup X_{3}\right) \\
& X_{3}=X_{2}+[1,1] \\
& X_{4}=X_{4} \nabla\left(X_{2} \sqcup X_{8} \sqcup X_{5} \sqcup X_{6}\right) \\
& X_{5}=X_{4}+[1,1] \\
& X_{6}=[0,0] \\
& X_{7}=X_{4} \\
& X_{8}=[1,1]
\end{aligned}
$$

(c)

Fig. 1. (a) A simple program $P$ that updates $x$; (b) Corresponding equation system for interval domain; (c) Corresponding equation system with vertices 2 and 4 as widening points.

Widening. For non-Noetherian abstract domains, such as the interval abstract domain, termination of this Kleene iteration sequence requires the use of a widening operator $(\nabla)$ [Cousot 2015; Cousot and Cousot 1977]. A set of widening points $W$ is chosen and the equation for $i \in W$ is replaced by $\mathcal{X}_{i}=\mathcal{X}_{i} \nabla F_{i}\left(\mathcal{X}_{0}, \ldots, \mathcal{X}_{n}\right)$. An admissible set of widening points "cuts" each cycle in the dependency graph of the equation system by the use of a widening operator to ensure termination [Cousot and Cousot 1977]. Finding a minimal admissible set of widening points is an NP-complete problem [Garey and Johnson 1979]. A possible widening operator for the interval abstract domain is defined by: $\perp \nabla I=I \nabla \perp=I \in$ Int and $[i, j] \nabla[k, l]=$ [if $k<i$ then $-\infty$ else $i$, if $l>j$ then $\infty$ else $j]$. This widening operator is non-monotone. The application of a widening operator may result in a crude over-approximation of the least fixpoint; more sophisticated widening operators as well as techniques such as narrowing can be used to ensure precision [Amato and Scozzari 2013; Amato et al. 2016; Cousot and Cousot 1977; Gopan and Reps 2006; Kim et al. 2016]. Although the discussion of our fixpoint algorithm uses a simple widening strategy (§5), our implementation incorporates more sophisticated widening and narrowing strategies implemented in IKOS (§8).

Bourdoncle's approach. Bourdoncle [1993] introduces the notion of hierarchical total order (HTO) of a set and weak topological order (WTO) of a directed graph (see §7). An admissible set of widening points as well as a chaotic iteration strategy, called the recursive strategy, can be computed using a WTO of the dependency graph of the equation system. A WTO for the equation system in Figure $1(\mathrm{~b})$ is $T \stackrel{\text { def }}{=} 081(23)(456)$ 7. The set of elements between two matching parentheses are called a component of the WTO, and the first element of a component is called the head of the component. Notice that components are non-trivial strongly connected components ("loops") in the directed graph of Figure 1(a). Bourdoncle [1993] proves that the set of component heads is an admissible set of widening points. For Figure 1(b), the set of heads $\{2,4\}$ is an admissible set of widening points. Figure 1(c) shows the corresponding equation system that uses widening.

The iteration strategy generated using WTO $T$ is $S_{1} \stackrel{\text { def }}{=} 081[23]^{*}\left[\begin{array}{ll}4 & 5\end{array}\right]^{*} 7$, where occurrence of $i$ in the sequence represents applying the equation for $\mathcal{X}_{i}$, and [... $]^{*}$ is the "iterate until stabilization" operator. A component is stabilized if iterating over its elements does not change their values. The component heads 2 and 4 are chosen as widening points. The iteration sequence $S_{1}$ should be interpreted as "apply equation for $\mathcal{X}_{0}$, then apply the equation for $\mathcal{X}_{8}$, then apply the equation for $\mathcal{X}_{1}$, repeatedly apply equations for $\mathcal{X}_{2}$ and $\mathcal{X}_{3}$ until stabilization" and so on. Furthermore, Bourdoncle [1993] showed that stabilization of a component can be detected by the stabilization of its head. For instance, stabilization of component $\{2,3\}$ can be detected by the stabilization of its head 2 . 
This property minimizes the number of (potentially expensive) comparisons between abstract values during fixpoint computation. For the equation system of Figure 1(c), the use of Bourdoncle's recursive iteration strategy would give us $X_{7}^{\mathrm{fp}}=[0, \infty]$.

Asynchronous iterations. The iteration strategy produced by Bourdoncle's approach is necessarily sequential, because the iteration sequence is generated from a total order. One could alternatively implement a parallel fixpoint computation using asynchronous iterations [Cousot 1977]: each processor $i$ computes the new value of $\mathcal{X}_{i}$ accessing the shared state consisting of $\mathcal{X}$ using the appropriate equation from Figure 1(c). However, the parallel fixpoint computation using asynchronous iterations is non-deterministic; that is, the fixpoint computed might differ based on the order in which the equations are applied (as noted by Monniaux [2005]). The reason for this non-determinism is due to the non-monotonicity of widening. For example, if the iteration sequence

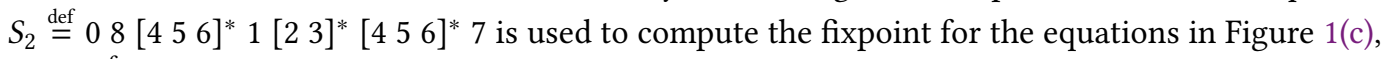
then $\mathcal{X}_{7}^{\mathrm{fp}}=[-\infty, \infty]$, which differs from the value computed using iteration sequence $S_{1}$.

Our deterministic parallel fixpoint computation. In this paper, we present a parallel fixpoint computation that is deterministic, and, in fact, gives the same result as Bourdoncle's sequential fixpoint computation (§7). Our approach generalizes Bourdoncle’s hierarchical total order and weak topological order to hierarchical partial order (HPO) and weak partial order (WPO) (§4). The iteration strategy is then based on the WPO of the dependency graph of the equation system. The use of partial orders, instead of total orders, enables us to generate an iteration strategy that is concurrent $(\S 5)$. For the equation system in Figure 1(c), our approach would produce the iteration sequence represented as $S_{3} \stackrel{\text { def }}{=} 0\left(\left(1\left[\begin{array}{ll}2 & 3\end{array}\right]^{*}\right) \mid 8\right)[4(5 \mid 6)]^{*} 7$, where | represents concurrent execution. Thus, the iteration (sub)sequences $1\left[\begin{array}{ll}2 & 3\end{array}\right]^{*}$ and 8 can be computed in parallel, as well as the subsequences 5 and 6. However, unlike iteration sequence $S_{2}$, the value for $\mathcal{X}_{4}$ in $S_{3}$ will not be computed until the component $\{2,3\}$ stabilizes. Intuitively, determinism is achieved by ensuring that no element outside the component will read the value of an element in the component until the component stabilizes. In our algorithm, the value of 2 is read by elements outside of the component $\{2,3\}$ only after the component stabilizes. Similarly, the value of 4 will be read by 7 only after the component $\{4,5,6\}$ stabilizes. Parallel fixpoint computation based on a WPO results in the same fixpoint as the sequential computation based on a WTO (§7).

\section{MATHEMATICAL PRELIMINARIES}

A binary relation $\mathrm{R}$ on set $S$ is a subset of the Cartesian product of $S$ and $S$; that is, $\mathrm{R} \subseteq S \times S$. Given $S^{\prime} \subseteq S$, let $\mathrm{R}_{\mid S^{\prime}}=\mathrm{R} \cap\left(S^{\prime} \times S^{\prime}\right)$. A relation $\mathrm{R}$ on set $S$ is said to be one-to-one iff for all $w, x, y, z \in S,(x, z) \in \mathrm{R}$ and $(y, z) \in \mathrm{R}$ implies $x=y$, and $(w, x) \in \mathrm{R}$ and $(w, y) \in \mathrm{R}$ implies $x=y$. A transitive closure of a binary relation $\mathrm{R}$, denoted by $\mathrm{R}^{+}$, is the smallest transitive binary relation that contains $\mathrm{R}$. A reflexive transitive closure of a binary relation $\mathrm{R}$, denoted by $\mathrm{R}^{*}$, is the smallest reflexive transitive binary relation that contains $R$.

A preorder $(S, \mathrm{R})$ is a set $S$ and a binary relation $\mathrm{R}$ over $S$ that is reflexive and transitive. A partial $\operatorname{order}(S, \mathrm{R})$ is a preorder where $\mathrm{R}$ is antisymmetric. Two elements $u, v \in S$ are comparable in a partial order $(S, \mathrm{R})$ if $(u, v) \in \mathrm{R}$ or $(v, u) \in \mathrm{R}$. A linear (total) order or chain is a partial order in which every pair of its elements are comparable. A partial order $\left(S, \mathrm{R}^{\prime}\right)$ is an extension of a partial order $(S, \mathrm{R})$ if $\mathrm{R} \subseteq \mathrm{R}^{\prime}$; an extension that is a linear order is called a linear extension. There exists a linear extension for every partial order [Szpilrajn 1930].

Given a partial order $(S, \mathrm{R})$, define $\lfloor x\rceil_{\mathrm{R}} \stackrel{\text { def }}{=}\{y \in S \mid(x, y) \in R\}$, and $\left\lfloor x \|_{\mathrm{R}} \stackrel{\text { def }}{=}\{v \in S \mid(v, x) \in R\}\right.$,

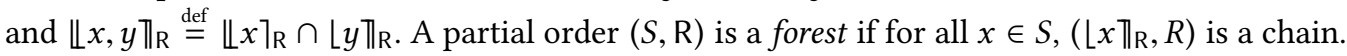


Example 3.1. Let $(Y, T)$ be a partial order with $Y=\left\{y_{1}, y_{2}, y_{3}, y_{4}\right\}$ and $\mathrm{T}=\left\{\left(y_{1}, y_{2}\right),\left(y_{2}, y_{3}\right)\right.$, $\left.\left(y_{2}, y_{4}\right)\right\}^{*}$. Let $Y^{\prime}=\left\{y_{1}, y_{2}\right\} \subseteq Y$, then $\mathrm{T}_{\left\lfloor Y^{\prime}\right.}=\left\{\left(y_{1}, y_{1}\right),\left(y_{1}, y_{2}\right),\left(y_{2}, y_{2}\right)\right\}$.

$$
\begin{aligned}
& \left.\left.\left.\left.\llbracket y_{1}\right\rceil_{\mathrm{T}}=\left\{y_{1}, y_{2}, y_{3}, y_{4}\right\} \quad \llbracket y_{2}\right\rceil_{\mathrm{T}}=\left\{y_{2}, y_{3}, y_{4}\right\} \quad \llbracket y_{3}\right\rceil_{\mathrm{T}}=\left\{y_{3}\right\} \quad \| y_{4}\right\rceil_{\mathrm{T}}=\left\{y_{4}\right\} \\
& \left\lfloor y_{1} \rrbracket_{\mathrm{T}}=\left\{y_{1}\right\} \quad\left\lfloor y_{2} \|_{\mathrm{T}}=\left\{y_{1}, y_{2}\right\} \quad\left\lfloor y_{3} \|_{\mathrm{T}}=\left\{y_{1}, y_{2}, y_{3}\right\} \quad\left\lfloor y_{4} \|_{\mathrm{T}}=\left\{y_{1}, y_{2}, y_{4}\right\}\right.\right.\right.\right.
\end{aligned}
$$

We see that the partial order $(Y, T)$ is a forest because for all $y \in Y,\left(\left\lfloor y \|_{\mathrm{T}}, \mathrm{T}\right)\right.$ is a chain.

$$
\begin{aligned}
& \left\|y_{1}, y_{1} \rrbracket_{\mathrm{T}}=\left\{y_{1}\right\} \quad\right\| y_{1}, y_{2} \rrbracket_{\mathrm{T}}=\left\{y_{1}, y_{2}\right\} \quad\left\|y_{1}, y_{3} \rrbracket_{\mathrm{T}}=\left\{y_{1}, y_{2}, y_{3}\right\} \quad\right\| y_{1}, y_{4} \pi_{\mathrm{T}}=\left\{y_{1}, y_{2}, y_{4}\right\} \\
& \left\|y_{4}, y_{1}\right\|_{\mathrm{T}}=\emptyset \quad \llbracket y_{4}, y_{2} \pi_{\mathrm{T}}=\emptyset \quad\left\|y_{4}, y_{3} \pi_{\mathrm{T}}=\emptyset \quad\right\| y_{4}, y_{4} \pi_{\mathrm{T}}=\left\{y_{4}\right\}
\end{aligned}
$$

A directed graph $G(V, \rightarrow)$ is defined by a set of vertices $V$ and a binary relation $\rightarrow$ over $V$. The reachability among vertices is captured by the preorder $\rightarrow^{*}:$ there is a path from vertex $u$ to vertex $v$ in $G$ iff $u \rightarrow{ }^{*} v$. $G$ is a directed acyclic graph $(D A G)$ iff $\left(V, \rightarrow^{*}\right)$ is a partial order. A topological order of a DAG $G$ corresponds to a linear extension of the partial order $\left(V, \rightarrow^{*}\right)$. We use $G_{\mid V^{\prime}}$ to denote the subgraph $\left(V \cap V^{\prime}, \rightarrow \mid V^{\prime}\right)$. Given a directed graph $G(V, \rightarrow)$, a depth-first numbering $(D F N)$ is the order in which vertices are discovered during a depth-first search (DFS) of G. A post depth-first numbering (post-DFN) is the order in which vertices are finished during a DFS of G. A depth-first tree (DFT) of $G$ is a tree formed by the edges used to discover vertices during a DFS. Given a DFT of $G$, an edge $u \rightarrow v$ is called (i) a tree edge if $v$ is a child of $u$ in the DFT; (ii) a back edge if $v$ is an ancestor of $u$ in the DFT; (iii) a forward edge if it is not a tree edge and $v$ is a descendant of $u$ in the DFT; and (iv) a cross edge otherwise [Cormen et al. 2009]. In general, a directed graph might contain multiple connected components and a DFS yields a depth-first forest (DFF). The lowest common ancestor (LCA) of vertices $u$ and $v$ in a rooted tree $T$ is a vertex that is an ancestor of both $u$ and $v$ and that has the greatest depth in $T$ [Tarjan 1979]. It is unique for all pairs of vertices.

A strongly connected component $(S C C)$ of a directed graph $G(V, \rightarrow)$ is a subgraph of $G$ such that $u \rightarrow^{*} v$ for all $u, v$ in the subgraph. An SCC is trivial if it only consists of a single vertex without any edges. A feedback edge set $B$ of a graph $G(V, \rightarrow)$ is a subset of $\rightarrow$ such that $\left(V,(\rightarrow \backslash B)^{*}\right)$ is a partial order; that is, the directed graph $G(V, \rightarrow \backslash B)$ is a DAG. The problem of finding the minimum feedback edge set is NP-complete [Karp 1972].

Example 3.2. Let $G(V, \rightarrow)$ be directed graph shown in Figure 1(a). The ids used to label the vertices $V$ of $G$ correspond to a depth-first numbering (DFN) of the directed graph $G$. The following lists the vertices in increasing post-DFN numbering: $3,5,6,7,4,2,1,8,0$. Edges $(3,2),(5,4)$, and $(6,4)$ are back edges for the DFF that is assumed by the DFN, edge $(8,4)$ is a cross edge, and the rest are tree edges. The lowest common ancestor (LCA) of 3 and 7 in this DFF is 2 . The subgraphs induced by the vertex sets $\{2,3\},\{4,5\},\{4,6\}$, and $\{4,5,6\}$ are all non-trivial SCCs. The minimum feedback edge set of $G$ is $F=\{(3,2),(5,4),(6,4)\}$. We see that the graph $G(V, \rightarrow \backslash F)$ is a DAG.

\section{AXIOMATIC CHARACTERIZATION OF WEAK PARTIAL ORDER}

This section introduces the notion of Weak Partial Order (WPO), presents its axiomatic characterization, and proves relevant properties. A constructive characterization is deferred to $\S 6$. The notion of WPO is built upon the notion of a hierarchical partial order, which we define first.

A Hierarchical Partial Order (HPO) is a partial order $(S, \preceq)$ overlaid with a nesting relation $\mathrm{N} \subseteq S \times S$ that structures the elements of $S$ into well-nested hierarchical components. As we will see in $\S 5$, the elements in a component are iterated over until stabilization in a fixpoint iteration strategy, and the partial order enables concurrent execution. 
Definition 4.1. A hierarchical partial $\operatorname{order} \mathcal{H}$ is a 3-tuple $(S, \leq, \mathrm{N})$ such that:

H1. $(S, \preceq)$ is a partial order.

H2. $\mathrm{N} \subseteq S \times S$ is one-to-one.

H3. $(x, h) \in \mathrm{N}$ implies $h<x$.

H4. Partial order $\left(C_{\mathcal{H}}, \subseteq\right)$ is a forest, where $C_{\mathcal{H}} \stackrel{\text { def }}{=}\left\{\left\lfloor h, x \rrbracket_{\leq} \mid(x, h) \in \mathrm{N}\right\}\right.$ is the set of components. H5. For all $h, x, u, v \in S, h \leq u \leq x$ and $(x, h) \in \mathrm{N}$ and $u \leq v$ implies either $x<v$ or $v \leq x$.

For each $(x, h) \in \mathrm{N}$, the set $\llbracket h, x \rrbracket_{\leq} \stackrel{\text { def }}{=}\{u \in S \mid h \leq u \leq x\}$ defines a component of the HPO, with $x$ and $h$ referred to as the exit and head of the component. A component can be identified using either its head or its exit due to condition $\mathrm{H} 2$; we use $C_{h}$ or $C_{x}$ to denote a component with head $h$ and exit $x$. Condition $\mathrm{H} 3$ states that the nesting relation $\mathrm{N}$ is in the opposite direction of the partial order $\leq$. The reason for this convention will be clearer when we introduce the notion of WPO (Definition 4.3), where we show that the nesting relation $N$ has a connection to the feedback edge set of the directed graph. Condition $\mathrm{H} 4$ implies that the set of components $C_{\mathcal{H}}$ is well-nested; that is, two components should be either mutually disjoint or one must be a subset of the other.

Condition $\mathrm{H} 5$ states that if an element $v$ depends upon an element $u$ in a component $C_{x}$, then either $v$ depends on the exit $x$ or $v$ is in the component $C_{x}$. Recall that $(x, h) \in \mathrm{N}$ and $h \leq u \leq x$ implies $u \in C_{x}$ by definition. Furthermore, $v \leq x$ and $u \leq v$ implies $v \in C_{x}$. Condition H5 ensures determinism of the concurrent iteration strategy ( $(5)$; this condition ensures that the value of $u$ does not "leak" from $C_{x}$ during fixpoint computation until the component $C_{x}$ stabilizes.

Example 4.2. Consider the partial order $(Y, \mathrm{~T})$ defined in Example 3.1. Let $\mathrm{N}_{1}=\left\{\left(y_{3}, y_{1}\right),\left(y_{4}, y_{2}\right)\right\}$. $\left(Y, \mathrm{~T}, \mathrm{~N}_{1}\right)$ violates condition $\mathrm{H} 4$. In particular, the components $C_{y_{3}}=C_{y_{1}}=\left\{y_{1}, y_{2}, y_{3}\right\}$ and $C_{y_{4}}=$ $C_{y_{2}}=\left\{y_{2}, y_{4}\right\}$ are neither disjoint nor is one a subset of the other. Thus, $\left(Y, \mathrm{~T}, \mathrm{~N}_{1}\right)$ is not an HPO.

Let $\mathrm{N}_{2}=\left\{\left(y_{3}, y_{1}\right)\right\}$. $\left(Y, \mathrm{~T}, \mathrm{~N}_{2}\right)$ violates condition H5. In particular, $y_{2} \in C_{y_{3}}$ and $\left(y_{2}, y_{4}\right) \in \mathrm{T}$, but we do not have $y_{3} \prec y_{4}$ or $y_{4} \leq y_{3}$. Thus, $\left(Y, \mathrm{~T}, \mathrm{~N}_{2}\right)$ is not an HPO.

Let $\mathrm{N}_{3}=\left\{\left(y_{2}, y_{1}\right)\right\} .\left(Y, \mathrm{~T}, \mathrm{~N}_{3}\right)$ is an HPO satisfying all conditions $\mathrm{H} 1-\mathrm{H} 5$.

Building upon the notion of an HPO, we now define a Weak Partial Order (WPO) for a directed graph $G(V, \rightarrow)$. In the context of fixpoint computation, $G$ represents the dependency graph of the fixpoint equation system. To find an effective iteration strategy, the cyclic dependencies in $G$ need to be broken. In effect, a WPO partitions the preorder $\rightarrow^{*}$ into a partial order $\rightarrow^{*}$ and an edge set defined using of a nesting relation $\rightarrow$.

Definition 4.3. A weak partial order $\mathcal{W}$ for a directed graph $G(V, \rightarrow)$ is a 4-tuple $(V, X, \rightarrow,-\rightarrow)$ such that:

W1. $V \cap X=\emptyset$.

W2. $\rightarrow \subseteq X \times V$, and for all $x \in X$, there exists $v \in V$ such that $x \rightarrow v$.

W3. $\rightarrow \subseteq(V \cup X) \times(V \cup X)$.

W4. $\mathcal{H}\left(V \cup X, \rightarrow^{*},-\rightarrow\right)$ is a hierarchical partial order (HPO).

W5. For all $u \rightarrow v$, either (i) $u \rightarrow^{+} v$, or (ii) $u \in\left\lfloor v, x \|_{\rightarrow^{*}}\right.$ and $x \rightarrow v$ for some $x \in X$.

Condition W4 states that $\mathcal{H}\left(V \cup X, \rightarrow^{*},-\rightarrow\right)$ is an HPO. Consequently, $\left(V \cup X, \rightarrow^{*}\right)$ is a partial order and $\rightarrow$ plays the role of the nesting relation in Definition 4.1. We refer to the relation $\rightarrow$ in WPO $\mathcal{W}$ as the scheduling constraints, the relation $\rightarrow$ as stabilization constraints, and the set $X$ as the exits. Furthermore, the notion of components $C_{\mathcal{H}}$ of an HPO $\mathcal{H}$ as defined in Definition 4.1 can be lifted to components of WPO $C_{\mathcal{W}} \stackrel{\text { def }}{=}\left\{\left\lfloor h, x \|_{\rightarrow^{*}} \mid x \rightarrow h\right\}\right.$. Condition W4 ensures that the concurrent iteration strategy for the WPO is deterministic (§5). Condition W1 states that exits are always new. Condition W2 states that $X$ does not contain any unnecessary elements. 


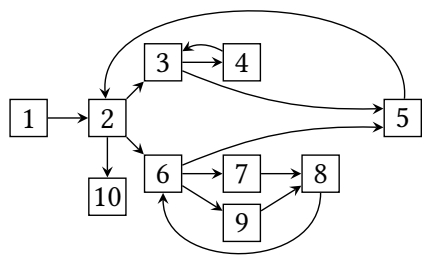

(a)

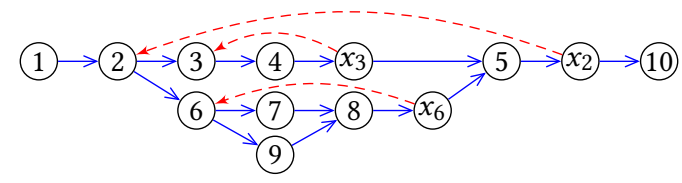

(b)

Fig. 2. (a) Directed graph $G_{1}$ and (b) WPO $\mathcal{W}_{1}$. Vertices $V$ are labeled using DFN; exits $X=\left\{x_{2}, x_{3}, x_{6}\right\}$.

Condition W5 connects the relation $\rightarrow$ of the directed graph $G$ with relations $\rightarrow$ and $\rightarrow$ used in the HPO $\mathcal{H}\left(V \cup X, \rightarrow^{*},-\rightarrow\right)$ in condition W4. Condition W5 ensures that all dependencies $u \rightarrow v$ in $G$ are captured by the HPO $\mathcal{H}$ either via a relation in the partial order $u \rightarrow^{+} v$ or indirectly via the component corresponding to $x \rightarrow v$, as formalized by the following theorem:

Theorem 4.4. For graph $G(V, \rightarrow)$ and its WPO $\mathcal{W}(V, X, \rightarrow,-\rightarrow), \rightarrow^{*} \subseteq(\rightarrow \cup-\rightarrow)^{*}$.

Proof. By property W5, for each $u \rightarrow v$, either $u \rightarrow^{+} v$, or $u \in\left\lfloor v, x \rrbracket_{\rightarrow^{*}}\right.$ and $x \rightarrow v$ for some

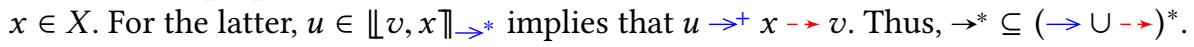

Example 4.5. Consider the directed graph $G_{1}(V, \rightarrow)$ in Figure 2(a). Figure 2(b) shows a WPO $\mathcal{W}_{1}(V, X, \rightarrow,-\rightarrow)$ for $G_{1}$, where $X=\left\{x_{2}, x_{3}, x_{6}\right\}$, and satisfies all conditions in Definition 4.3. One can verify that $\left(V \cup X, \rightarrow^{*},-\rightarrow\right)$ satisfies all conditions in Definition 4.1 and is an HPO.

Suppose we were to remove $x_{6} \rightarrow 5$ and instead add $6 \rightarrow 5$ to $\mathcal{W}_{1}$ (to more closely match the edges in $G_{1}$ ), then this change would violate condition $\mathrm{H} 5$, and hence condition $\mathrm{W} 4$.

If we were to only remove $x_{6} \rightarrow 5$ from $\mathcal{W}_{1}$, then it would still satisfy condition W4. However, this change would violate condition W5.

Definition 4.6. For graph $G(V, \rightarrow)$ and its WPO $\mathcal{W}(V, X, \rightarrow,-\rightarrow)$, the back edges of $G$ with respect to the $W P O \mathcal{W}$, denote by $B_{\mathcal{W}}$, are defined as $B_{\mathcal{W}} \stackrel{\text { def }}{=}\left\{(u, v) \in \rightarrow \mid \exists x \in X . u \in \llbracket v, x \|_{\left.\rightarrow^{*} \wedge x \rightarrow v\right\}}\right.$.

In other words, $(u, v) \in B_{\mathcal{W}}$ if $u \rightarrow v$ satisfies condition W5-(ii) in Definition 4.3. Theorem 4.7 proves that $B_{\mathcal{W}}$ is a feedback edge set for $G$, and Theorem 4.9 shows that the subgraph $\left(V, \rightarrow \backslash B_{\mathcal{W}}\right)$ forms a DAG. Together these two theorems capture the fact that the WPO $\mathcal{W}(V, X, \rightarrow,-\rightarrow)$ partitions the preorder $\rightarrow^{*}$ of $G(V, \rightarrow)$ into a partial order $\rightarrow^{*}$ and a feedback edge set $B_{W}$.

Theorem 4.7. For graph $G(V, \rightarrow)$ and its WPO $\mathcal{W}(V, X, \rightarrow,-\rightarrow), B_{\mathcal{W}}$ is a feedback edge set for $G$.

Proof. Let $v_{1} \rightarrow v_{2} \rightarrow \cdots \rightarrow v_{n} \rightarrow v_{1}$ be a cycle of $n$ distinct vertices in $G$. We will show that there exists $i \in[1, n)$ such that $v_{i} \rightarrow v_{i+1} \in B_{\mathcal{W}}$; that is, $v_{i} \in\left\|v_{i+1}, x\right\|_{\rightarrow^{*}}$ and $x \rightarrow v_{i+1}$ for some $x \in X$. If this were not true, then $v_{1} \rightarrow^{+} \cdots \rightarrow^{+} v_{n} \rightarrow^{+} v_{1}$ (using W5). Therefore, $v_{1} \rightarrow^{+} v_{n}$ and $v_{n} \rightarrow^{+} v_{1}$, which contradicts the fact that $\rightarrow^{*}$ is a partial order. Thus, $B_{\mathcal{W}}$ cuts all cycles at least once and is a feedback edge set.

Example 4.8. For the graph $G_{1}(V, \rightarrow)$ in Figure $2\left(\right.$ a) and WPO $\mathcal{W}_{1}(V, X, \rightarrow,-\rightarrow)$ in Figure 2(b), $B_{\mathcal{W}_{1}}=\{(4,3),(8,6),(5,2)\}$. One can verify that $B_{\mathcal{W}_{1}}$ is a feedback edge set for $G_{1}$.

Theorem 4.9. For graph $G(V, \rightarrow)$ and its WPO $\mathcal{W}(V, X, \rightarrow,-\rightarrow),\left(\rightarrow \backslash B_{\mathcal{W}}\right)^{+} \subseteq \rightarrow^{+}$.

Proof. Each edge $(u, v) \in B_{\mathcal{W}}$ satisfies W5-(ii) by definition. Therefore, all edges in $\left(\rightarrow \backslash B_{\mathcal{W}}\right)$ must satisfy W5-(i). Thus, $u \rightarrow^{+} v$ for all edges $(u, v) \in\left(\rightarrow \backslash B_{\mathcal{W}}\right)$, and $\left(\rightarrow \backslash B_{\mathcal{W}}\right)^{*} \subseteq \rightarrow^{*}$. 
Given the tuple $\mathcal{W}(V, X, \rightarrow,-\rightarrow)$ and a set $S$, we use $\mathcal{W}_{l S}$ to denote the tuple $(V \cap S, X \cap$ $\left.S, \rightarrow_{\mid S}, \rightarrow \mid S\right)$. The following two theorems enable us to decompose a WPO into sub-WPOs enabling the use of structural induction when proving properties of WPOs.

Theorem 4.10. For graph $G(V, \rightarrow)$ and its $W P O \mathcal{W}(V, X, \rightarrow,-\rightarrow), \mathcal{W}_{l C}$ is a WPO for subgraph $G_{l C}$ for all $C \in C_{\mathcal{W}}$.

Proof. We show that $\mathcal{W}_{l C}$ satisfies all conditions $\mathrm{W} 1-\mathrm{W} 5$ in Definition 4.3 for all $C \in C_{\mathcal{W}}$. Conditions W1, W2, W3, W4-[H1, H2, H3, H4] trivially holds true.

[W4-H5] If $v \notin C, \mathrm{H} 5$ is true because $(u, v) \notin \rightarrow^{*}{ }_{l C}$. Else, $\mathrm{H} 5$ is still satisfied with $\rightarrow^{*}{ }{ }_{C}$.

[W5] We show that $u \rightarrow^{+} v$ implies $u \rightarrow{ }^{+} C_{C} v$, if $u, v \in C$. Let $C=\left\lfloor h, x \|_{\rightarrow^{*}}\right.$ with $x \rightarrow h$. If $u \rightarrow^{+} \mid C v$ is false, then there exists $w \in\lfloor u\rceil_{\rightarrow^{*}} \cap\left\lfloor v \prod_{\rightarrow^{*}}\right.$ such that $w \notin C$. However, $u \rightarrow^{+} w$ and $w \notin C$ implies $x \rightarrow^{+} w$ (using H5). This contradicts that $\left(V \cup X, \rightarrow^{*}\right)$ is a partial order, because $w \in\left\lfloor v \|_{\rightarrow^{*}}\right.$ and $v \in \| h, x \rrbracket_{\rightarrow^{*}}$ implies $w \rightarrow^{+} x$. Thus, W5 is satisfied.

Theorem 4.11. For graph $G(V, \rightarrow)$ and its $W P O \mathcal{W}(V, X, \rightarrow,-\rightarrow)$, if $V \cup X=\left\lfloor h, x \rrbracket_{\rightarrow *}\right.$ for some $(x, h) \in \rightarrow$, then $\mathcal{W}_{\mid S}$ is a WPO for subgraph $G_{\mid S}$, where $S \stackrel{\text { def }}{=} V \cup X \backslash\{h, x\}$.

Proof. We show that $\mathcal{W}_{\lfloor S}$ satisfies all conditions W1-W5 in Definition 4.3. Conditions W1, W2, W3, W4-[H1, H2, H3, H4] trivially holds true.

[W4-H5] $x$ has no outgoing and $h$ has no incoming scheduling constraints. Thus, $\mathcal{W}_{l S}$ still satisfies H5.

[W5] Case (i) is still satisfied because $h$ only had outgoing scheduling constraints and $x$ only had incoming scheduling constraints. Case (ii) is still satisfied due to $\mathrm{H} 2$ and H4.

Example 4.12. The decomposition of WPO $\mathcal{W}_{1}$ for graph $G_{1}$ in Figure 2 is:

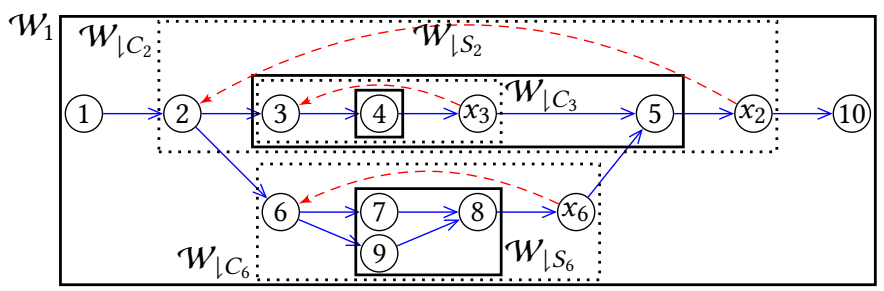

$C_{\mathcal{W}}=\left\{C_{2}, C_{3}, C_{6}\right\}$, where $C_{2}=\left\{2,3,4, x_{3}, 5, x_{2}\right\}, C_{3}=\left\{3,4, x_{3}\right\}$, and $C_{6}=\left\{6,7,8,9, x_{6}\right\}$. As proved in Theorem 4.10, $\mathcal{W}_{\mid C_{2}}, \mathcal{W}_{l_{C_{3}}}$, and $\mathcal{W}_{\mid C_{6}}$ (shown using dotted lines) are WPOs for the subgraphs $G_{\mid C_{2}}, G_{\mid C_{3}}$, and $G_{\mid C_{6}}$, respectively. Furthermore, Theorem 4.11 is applicable to each of these WPOs. Therefore, $\mathcal{W}_{l S_{2}}, \mathcal{W}_{l S_{3}}$, and $\mathcal{W}_{l S_{6}}$ (shown using solid lines) are WPOs for subgraphs $G_{\mid S_{2}}, G_{\mid S_{3}}$, and $G_{\left\lfloor S_{6}\right.}$, respectively, where $S_{h}=C_{h} \backslash\left\{h, x_{h}\right\}$ for $h \in\{2,3,6\}$. For example, $S_{6}=C_{6} \backslash\left\{6, x_{6}\right\}=\{7,8,9\}$. Note that $\mathcal{W}_{l C_{6}}$ is a WPO for subgraph 68 , while $\mathcal{W}_{l S_{6}}$ is a WPO for subgraph $\frac{7}{9} \rightarrow$

Definition 4.13. Given a WPO $\mathcal{W}, C \in C_{\mathcal{W}}$ is a maximal component if there does not exist another component $C^{\prime} \in C_{\mathcal{W}}$ such that $C \subset C^{\prime} . C_{\mathcal{W}}^{0}$ denotes the set of maximal components of $\mathcal{W}$.

Theorem 4.14. For graph $G(V, \rightarrow)$ and its $W P O \mathcal{W}(V, X, \rightarrow,-\rightarrow)$, if there is a cycle in $G$ consisting of vertices $V^{\prime}$, then there exists $C \in C_{\mathcal{W}}^{0}$ such that $V^{\prime} \subseteq C$.

Proof. Assume that the theorem is false. Then, there exists multiple maximal components that partition the vertices in the cycle. Let $(u, v)$ be an edge in the cycle where $u$ and $v$ are in different maximal components. By W5, $u \rightarrow^{+} v$, and by H5, $x_{u} \rightarrow^{+} v$, where $x_{u}$ is the exit of the maximal 


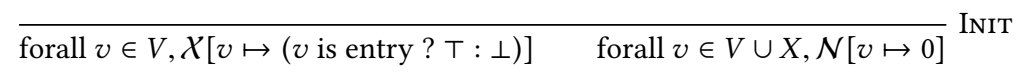

$$
\begin{aligned}
& \frac{v \in V \quad \mathcal{N}(v)=\operatorname{NumSchedPreds}(v)}{\mathcal{N}[v \mapsto 0] \quad \operatorname{Apply} F(v) \quad \text { forall } v \rightarrow w, \mathcal{N}[w \mapsto(\mathcal{N}[w]+1)]} \text { NonExit } \\
& \frac{x \in X \quad \mathcal{N}(x)=\operatorname{NumSchedPreds}(x) \quad \text { ComponentStabilized }(x)}{\mathcal{N}[x \mapsto 0] \quad \text { forall } x \rightarrow w, \mathcal{N}[w \mapsto(\mathcal{N}(w)+1)]} \text { CompStabilized } \\
& \frac{x \in X \quad \mathcal{N}(x)=\text { NumSchedPreds }(x) \quad \neg \text { ComponentStabilized }(x)}{\mathcal{N}[x \mapsto 0] \quad \text { SetNForComponent }(x)} \operatorname{CompNotStabILIZED} \\
& \operatorname{Apply} F(v) \stackrel{\text { def }}{=} X\left[v \mapsto\left(v \in \text { image of } \rightarrow \text { ? } \mathcal{X}(v) \nabla F_{v}(X): F_{v}(X)\right)\right] \\
& \text { ComponentStabilized }(x) \stackrel{\text { def }}{=} \exists h \in V \cdot x \rightarrow h \wedge F_{h}(X) \sqsubseteq \mathcal{X}(h) \\
& \text { SetNForComponent }(x) \stackrel{\text { def }}{=} \text { forall } v \in C_{x}, \mathcal{N}[v \mapsto \operatorname{NumOuterSchedPreds}(v, x)] \\
& \operatorname{NumSchedPreds}(v) \stackrel{\text { def }}{=}|\{u \in V \cup X \mid u \rightarrow v\}| \\
& \text { NumOuterSchedPreds }(v, x) \stackrel{\text { def }}{=}\left|\left\{u \in V \cup X \mid u \rightarrow v, u \notin C_{x}, v \in C_{x}\right\}\right|
\end{aligned}
$$

Fig. 3. Deterministic concurrent fixpoint algorithm for WPO. $\mathcal{X}$ maps an element in $V$ to its value. $\mathcal{N}$ maps an element in $V \cup X$ to its count of executed scheduling predecessors. Operations on $\mathcal{N}$ are atomic.

component that contains $u$. By the definition of the component, $v \rightarrow^{+} x_{v}$, where $x_{v}$ is the exit of the maximal component that contains $v$. Therefore, $x_{u} \rightarrow^{+} x_{v}$. Applying the same reasoning for all such edges in the cycle, we get $x_{u} \rightarrow^{+} x_{v} \rightarrow^{+} \cdots \rightarrow^{+} x_{u}$. This contradicts the fact that $\left(V \cup X, \rightarrow^{*}\right)$ is a partial order for the WPO $\mathcal{W}$.

Corollary 4.15. For $G(V, \rightarrow)$ and its WPOW $(V, X, \rightarrow,-\rightarrow)$, if $G$ is a non-trivial strongly connected graph, then there exists $h \in V$ and $x \in X$ such that $C_{\mathcal{W}}^{0}=\left\{\left\lfloor h, x \rrbracket_{\rightarrow^{*}}\right\}\right.$ and $\llbracket h, x \rrbracket_{\rightarrow^{*}}=V \cup X$.

Proof. Because there exists a cycle in the graph, there must exists at least one component in the WPO. Let $h \in V$ and $x \in X$ be the head and exit of a maximal component in $\mathcal{W}$. Because $V \cup X$ contains all elements in the WPO, $\left\lfloor h, x \rrbracket_{\rightarrow^{*}} \subseteq V \cup X\right.$. Now, suppose $\llbracket h, x \|_{\rightarrow^{*}} \nsupseteq V \cup X$. Then, there exists $v \in V \cup X$ such that $v \notin \llbracket h, x \|_{\rightarrow^{*}}$. If $v \in V$, then there exists a cycle $v \rightarrow^{+} h \rightarrow^{+} v$, because the graph is strongly connected. Then, by Theorem $4.14, v \in \llbracket h, x \|_{\rightarrow^{*}}$, which is a contradiction. If

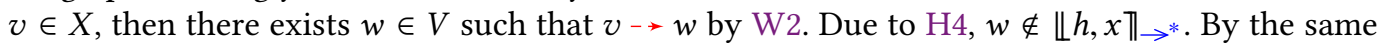
reasoning as the previous case, this leads to a contradiction.

\section{DETERMINISTIC CONCURRENT FIXPOINT ALGORITHM}

This section describes a deterministic concurrent algorithm for computing a fixpoint of an equation system. Given the equation system $\mathcal{X}=F(\mathcal{X})$ with dependency graph $G(V, \rightarrow)$, we first construct a WPO $\mathcal{W}(V, X, \rightarrow,-\rightarrow)$. The algorithm in Figure 3 uses $\mathcal{W}$ to compute the fixpoint of $\mathcal{X}=F(\mathcal{X})$. It defines a concurrent iteration strategy for a WPO: equations are applied concurrently by following the scheduling constraints $\rightarrow$, while stabilization constraints $\rightarrow$ act as "iterate until stabilization" operators, checking the stabilization at the exits and iterating the components.

Except for the initialization rule INIT, which is applied once at the beginning, rules in Figure 3 are applied concurrently whenever some element in $V \cup X$ satisfies the conditions. The algorithm uses a value map $\mathcal{X}$, which maps an element in $V$ to its abstract value, and a count map $\mathcal{N}$, which maps an element in $V \cup X$ to its counts of executed scheduling predecessors. Access to the value 
map $\mathcal{X}$ is synchronized by scheduling constraints, and operations on $\mathcal{N}$ are assumed to be atomic. Rule INIT initializes values for elements in $V$ to $\perp$ except for the entry of the graph, whose value is initialized to $T$. The counts for elements in $V \cup X$ are all initialized to 0 .

Rule NonExit applies to a non-exit element $v \in V$ whose scheduling predecessors are all executed $(\mathcal{N}(v)=$ NumSchedPreds $(v))$. This rule applies the function $F_{v}$ to update the value $X_{v}$ $(\operatorname{Apply} F(v))$. Definition of the function Apply $F$ shows that the widening is applied at the image of $\rightarrow$ (see Theorem 5.3). The rule then notifies the scheduling successors of $v$ that $v$ has executed by incrementing their counts. Because elements within a component can be iterated multiple times, the count of an element is reset after its execution. If there is no component in the WPO, then only the NonExit rule is applicable, and the algorithm reduces to a DAG scheduling algorithm.

Rules CompStabilized and CompNotStabilized are applied to an exit $x(x \in X)$ whose scheduling predecessors are all executed $(\mathcal{N}(x)=$ NumSchedPreds $(x))$. If the component $C_{x}$ is stabilized, CompStabilized is applied, and СомpNotStabilized otherwise. A component is stabilized if iterating it once more does not change the values of elements inside the component. Boolean function ComponentStabilized checks the stabilization of $C_{x}$ by checking the stabilization of its head (see Theorem 5.5). Upon stabilization, rule COMPSTABILIzED notifies the scheduling successors of $x$ and resets the count for $x$.

Example 5.1. An iteration sequence generated by rules in Figure 3 for WPO $\mathcal{W}_{1}$ (Figure 2(b)) is:

\begin{tabular}{c|cccccccccccccc}
\multicolumn{10}{l|}{} & Time step in $\mathbb{N} \longrightarrow$ \\
\hline Scheduled element & 1 & 2 & 3 & 4 & $x_{3}$ & 3 & 4 & $x_{3}$ & 3 & 4 & $x_{3}$ & 5 & $x_{2}$ & 10 \\
$u \in V \cup X$ & & & 6 & 7 & 8 & $x_{6}$ & 6 & 7 & 8 & $x_{6}$ & & & & \\
& & & & 9 & & & & & 9 & & &
\end{tabular}

The initial value of $\mathcal{N}(8)$ is 0 . Applying NonExit to 7 and 9 increments $\mathcal{N}(8)$ to 2 . $\mathcal{N}(8)$ now equals NumSchedPreds $(8)$, and NonExit is applied to 8 . Applying NonExit to 8 updates $\mathcal{X}_{8}$ by applying the function $F_{8}$, increments $\mathcal{N}\left(x_{6}\right)$, and resets $\mathcal{N}(8)$ to 0 . Due to the reset, same thing happens when $C_{6}$ is iterated once more. The initial value of $\mathcal{N}\left(x_{6}\right)$ is 0 . Applying NonExit to 8 increments $\mathcal{N}\left(x_{6}\right)$ to 1 , which equals NumSchedPreds $\left(x_{6}\right)$. The stabilization of component $C_{6}$ is checked at $x_{6}$. If it is stabilized, CompSTABILIzed is applied to $x_{6}$, which increments $\mathcal{N}(5)$ and resets $\mathcal{N}\left(x_{6}\right)$ to 0 .

The rule CompNotStabilized is applied if the component $C_{x}$ is not stabilized. Unlike rule CompStabilized, CompNotStabilized does not notify the scheduling successors of $x$. It resets the counts for each element in $C_{x}$ as well as that for $x$. SetNForComponent $(x)$ sets the count for $v \in C_{x}$ to NumOuterSchedPreds $(v, x)$, which is the number of its scheduling predecessors not in $C_{x}$. In particular, the count for the head of $C_{x}$, whose scheduling predecessors are all not in $C_{x}$, is set to the number of all scheduling predecessors, allowing rule NonExit to be applied to the head. The map NumOuterSchedPreds $(v, x)$ can be computed during WPO construction.

Example 5.2. Let $G_{2}$ be iteration sequence generated by the concurrent fixpoint algorithm for WPO $\mathcal{W}_{2}$ is:

\begin{tabular}{c|rrrrlllllllllll}
\multicolumn{10}{c|}{} & Time step in $\mathbb{N} \longrightarrow$ \\
\hline Scheduled element & 1 & 2 & 3 & 4 & $x_{3}$ & 3 & 4 & $x_{3}$ & $x_{2}$ & 2 & 3 & 4 & $x_{3}$ & $x_{2}$ \\
$u \in V \cup X$ & & 6 & 5 & & & & & & & & 5 & & &
\end{tabular}

Consider the element 4, whose scheduling predecessors in the WPO are 3, 5, and 6. Furthermore, $4 \in C_{3}$ and $4 \in C_{2}$ with $C_{3} \subsetneq C_{2}$. After NonExit is applied to $4, \mathcal{N}(4)$ is reset to 0 . Then, if the stabilization check of $C_{3}$ fails at $x_{3}$, CompNotStabilized sets $\mathcal{N}(4)$ to 2 , which is 
NumOuterSchedPreds $\left(4, x_{3}\right)$. If it is not set to 2 , then the fact that elements 5 and 6 are executed will not be reflected in $\mathcal{N}(4)$, and the iteration over $C_{3}$ will be blocked at element 4 . If the stabilization check of $C_{2}$ fails at $x_{2}$, COMPNotStabilized sets $\mathcal{N}(4)$ to NumOuterSchedPreds $\left(4, x_{2}\right)=1$.

In ApplyF, the image of $\rightarrow$ is chosen as the set of widening points. These are heads of the components. The following theorem proves that the set of component heads is an admissible set of the widening points, which guarantee the termination of the fixpoint computation:

Theorem 5.3. Given a dependency graph $G(V, \rightarrow)$ and its WPO $\mathcal{W}(V, X, \rightarrow,-\rightarrow)$, the set of component heads is an admissible set of widening points.

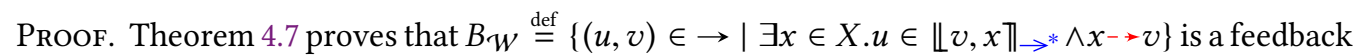
edge set. Consequently, the set of component heads $\{h \mid \exists x \in X . x \rightarrow h\}$ is a feedback vertex set. Therefore, the set $W$ is an admissible set of widening points [Cousot and Cousot 1977].

Example 5.4. The set of component heads $\{2,3,6\}$ is an admissible set of widening points for the WPO $\mathcal{W}_{1}$ in Figure 2(b).

The following theorem justifies our definition of ComponentStabilized; viz., checking the stabilization of $\mathcal{X}_{h}$ is sufficient for checking the stabilization of the component $C_{h}$.

THEOREM 5.5. During the execution of concurrent fixpoint algorithm with WPO $\mathcal{W}(V, X, \rightarrow,-\rightarrow)$, stabilization of the head $h$ implies the stabilization of the component $C_{h}$ at its exit for all $C_{h} \in C_{\mathcal{W}}$.

Proof. Suppose that there exists an element $v \in C_{h}$ such that $v$ is not stabilized although the head $h$ is stabilized. That is, suppose that $\mathcal{X}_{v}$ changes when $C_{h}$ is iterated once more. For this to be possible, there must exist $u \in V$ such that $u \rightarrow v$ and $\mathcal{X}_{u}$ changed after the last update of $\mathcal{X}_{v}$. By $u \rightarrow v$ and W5, either (i) $u \rightarrow^{+} v$ or (ii) $u \in\|v, x\|_{\rightarrow^{*}}=C_{v}$ and $x \rightarrow v$ for some $x \in X$. It cannot be the case (i), because $u \rightarrow^{+} v$ and H5 imply that $\mathcal{X}_{u}$ cannot be updated after the last update of $\mathcal{X}_{v}$. Therefore, it should always be the case (ii). By H4, $C_{v} \subsetneq C_{h}$. However, because $u \in C_{v}$, our algorithm checks the stabilization of $v$ at the exit of $C_{v}$ after the last update of $\mathcal{X}_{u}$. This contradicts the assumption that $\mathcal{X}_{u}$ changed after the last update of $\mathcal{X}_{v}$.

A WPO $\mathcal{W}(V, X, \rightarrow,-\rightarrow)$ where $V=\{v\}, X=\rightarrow=-\rightarrow=\emptyset$ is said to be a trivial $W P O$, which is represented as $(0$. It can only be a WPO for a trivial SCC with vertex $v$. A WPO $\mathcal{W}(V, X, \rightarrow,-\rightarrow)$ where $V=\{h\}, X=\{x\}, \rightarrow=\{(h, x)\},-\rightarrow=(x, h)$ is said to be a self-loop WPO, and is represented as $(h) \rightarrow$. It can only be a WPO for a trivial SCC with vertex $h$ or a single vertex $h$ with a self-loop. The following theorem proves that the concurrent fixpoint algorithm in Figure 3 is deterministic.

Theorem 5.6. Given a WPO $\mathcal{W}(V, X, \rightarrow,-\rightarrow)$ for a graph $G(V, \rightarrow)$ and a set of monotonone, deterministic functions $\left\{F_{v} \mid v \in V\right\}$, concurrent fixpoint algorithm in Figure 3 is deterministic, computing the same approximation of the least fixpoint for the given set of functions.

Proof. We use structural induction on the WPO $\mathcal{W}$ to show this. [BASE CASE]: The two cases for the base case are (i) $\mathcal{W}=$ (v) and (ii) $\mathcal{W}=(h>x$. If $\mathcal{W}=$ (v), then $v$ is the only vertex in $G$. Functions are assumed to be deterministic, so applying the function $F_{v}()$ in rule NonExit of Figure 3 is deterministic. Because $F_{v}()$ does not take any arguments, the computed value $\mathcal{X}_{v}$ is a unique fixpoint of $F_{v}()$.

If $\mathcal{W}=(h)\left(x\right.$, then $h$ is the only vertex in $G$. If $h$ has a self-loop, function $F_{h}\left(X_{h}\right)$ and widening operator $\nabla$ may need to be applied multiple times to reach a post-fixpoint (approximation of the least fixpoint) of $F_{h}\left(X_{h}\right)$. Rule NonExit applies them once on $X_{h}$ and signals the exit $x$. If the post-fixpoint of $F_{h}\left(X_{h}\right)$ is not reached, ComponentStabilized returns false, and rule CompNotStabilized in Figure 3 applies rule NonExit on $h$ again. If the post-fixpoint is reached, ComponentStabilized 
returns true, and rule CompSTABILIzED in Figure 3 stops the algorithm. Because $F_{h}\left(\mathcal{X}_{h}\right)$ and $\nabla$ are deterministic, each iteration is deterministic, and the entire sequence of iterations are deterministic. The computed value $\mathcal{X}_{h}$ is a post-fixpoint of $F_{h}\left(X_{h}\right)$.

[INDUCTIVE STEP]: By condition H4 and Theorem 4.10, $\mathcal{W}$ can be decomposed into a set of WPOs of its maximal components and trivial WPOs. The two cases for the inductive step are (i) the decomposition of $\mathcal{W}$ is a single $\mathrm{WPO}$ of the maximal component and (ii) $\mathcal{W}$ is decomposed into multiple WPOs.

If the decomposition of $\mathcal{W}$ is a single WPO of the maximal component $\llbracket h, x \rrbracket_{\rightarrow^{*}}$, then by

Theorem 4.11, $\mathcal{W}=h\left(\mathcal{W}_{l S} x\right.$, where $S \stackrel{\text { def }}{=}\left\lfloor h, x \|_{\rightarrow^{*}} \backslash\{h, x\}\right.$ and $\mathcal{W}_{l S}$ is a WPO. By the induction hypothesis, the fixpoint algorithm is deterministic for $\mathcal{W}_{l S}$. The head $h$ of $\mathcal{W}$ is its unique source, so each iteration begins with the application of rule NonExiT on $h$. After applying $F_{h}(\cdot)$ and $\nabla$, rule NonExiT signals all its scheduling successors, initiating the iteration over $\mathcal{W}_{l S}$. Because all sinks of $\mathcal{W}_{l S}$ are connected to the exit $x$ of $\mathcal{W}, x$ will be processed after the iteration finishes. Thus, $\mathcal{X}_{h}$ remains fixed during the iteration. A single iteration over $\mathcal{W}_{\mid S}$ is identical to running the fixpoint algorithm on $\mathcal{W}_{l S}$ with the set of functions $\left\{F_{v}^{\prime} \mid v \in V^{\prime}\right\}$, where $\mathcal{W}_{l S}$ is a WPO for subgraph $G_{\mid S}$ and function $F_{v}^{\prime}$ is a partial application of $F_{v}$ that binds the argument that take $X_{h}$ to its current value. The number of functions and the arity of each function decrease. Because $F_{h}(\cdot)$ and $\nabla$ are deterministic, and each iteration over $\mathcal{W}_{l S}$ is deterministic, it is deterministic for $\mathcal{W}$. The algorithm iterates until the post-fixpoint of $F_{h}(\cdot)$ is reached, and by Theorem 5.5 , the computed value $X_{v}$ is a post-fixpoint of $F_{v}(\cdot)$ for all $v \in V$.

If $\mathcal{W}$ is decomposed into multiple WPOs, then by the induction hypothesis, the fixpoint algorithm deterministically computes the post-fixpoints for all sub-WPOs. Let $\mathcal{W}_{i}\left(V_{i}, X_{i}, \rightarrow\left|V_{i} \cup X_{i},-\rightarrow\right| V_{i} \cup X_{i}\right)$ be an arbitrary sub-WPO. For any $u \in V \backslash V_{i}$ such that $u \rightarrow v$, we have $u \rightarrow^{+} v$ by W5 and H4. Hence, $v$ is processed after $u$. Combined with $\mathrm{H}_{5}, \mathcal{X}_{u}$ remains unchanged during the iteration of $\mathcal{W}_{i}$. Single iteration over $\mathcal{W}_{i}$ is equal to running the fixpoint algorithm on $\mathcal{W}_{i}$ with the set of the functions $\left\{F_{v}^{\prime} \mid v \in V^{\prime}\right\}$, where function $F_{v}^{\prime}$ is a partial application of $F_{v}$ that binds the arguments in $V \backslash V_{i}$ to their current values. The number of functions decreases, and the arity of the functions does not increase. The outer scheduling predecessors of $W_{i}$ can be ignored in the iterations by SetNForComponent in rule CompNotSTABILIzED. Therefore, by the induction hypothesis, each iteration over $\mathcal{W}_{i}$ is deterministic, and because the choice of $\mathcal{W}_{i}$ is arbitrary, the algorithm is deterministic for $\mathcal{W}$. Furthermore, $(v, u) \notin \rightarrow^{+}$for any $u \in V \backslash V_{i}$ such that $u \rightarrow v$, because its negation would contradict Theorem 4.14. Therefore, change in $\mathcal{X}_{v}$ does not change $\mathcal{X}_{u}$, and $\mathcal{X}_{u}$ is still a post-fixpoint of $F_{u}(\cdot)$.

\section{ALGORITHMS FOR WPO CONSTRUCTION}

This section presents two algorithms for constructing a WPO for a graph $G(V, \rightarrow)$. The first algorithm, ConstructWPO ${ }^{\mathrm{TD}}$, is a top-down recursive algorithm that is inefficient but intuitive (§6.1). The second one, ConstructWPO ${ }^{\mathrm{BU}}$, is an efficient bottom-up iterative algorithm that has almostlinear time complexity (§6.2). Both algorithms do not introduce superfluous scheduling constraints that could restrict the parallelism during the fixpoint computation.

\subsection{Top-down Recursive Construction}

Algorithm 1 presents a top-down recursive algorithm ConstructWPO ${ }^{\mathrm{TD}}$, which acts as a proxy between the axiomatic characterization of WPO in $\S 4$ and the efficient construction algorithm ConstructWPO $0^{\mathrm{BU}}$ in $\S 6.2$.

ConstructWPO ${ }^{\text {TD }}$ is parametrized by the depth-first forest (DFF) of the graph $G$, and it may yield a different WPO for a different DFF. ConstructWPO ${ }^{\mathrm{TD}}$ begins with the identification of the maximal 


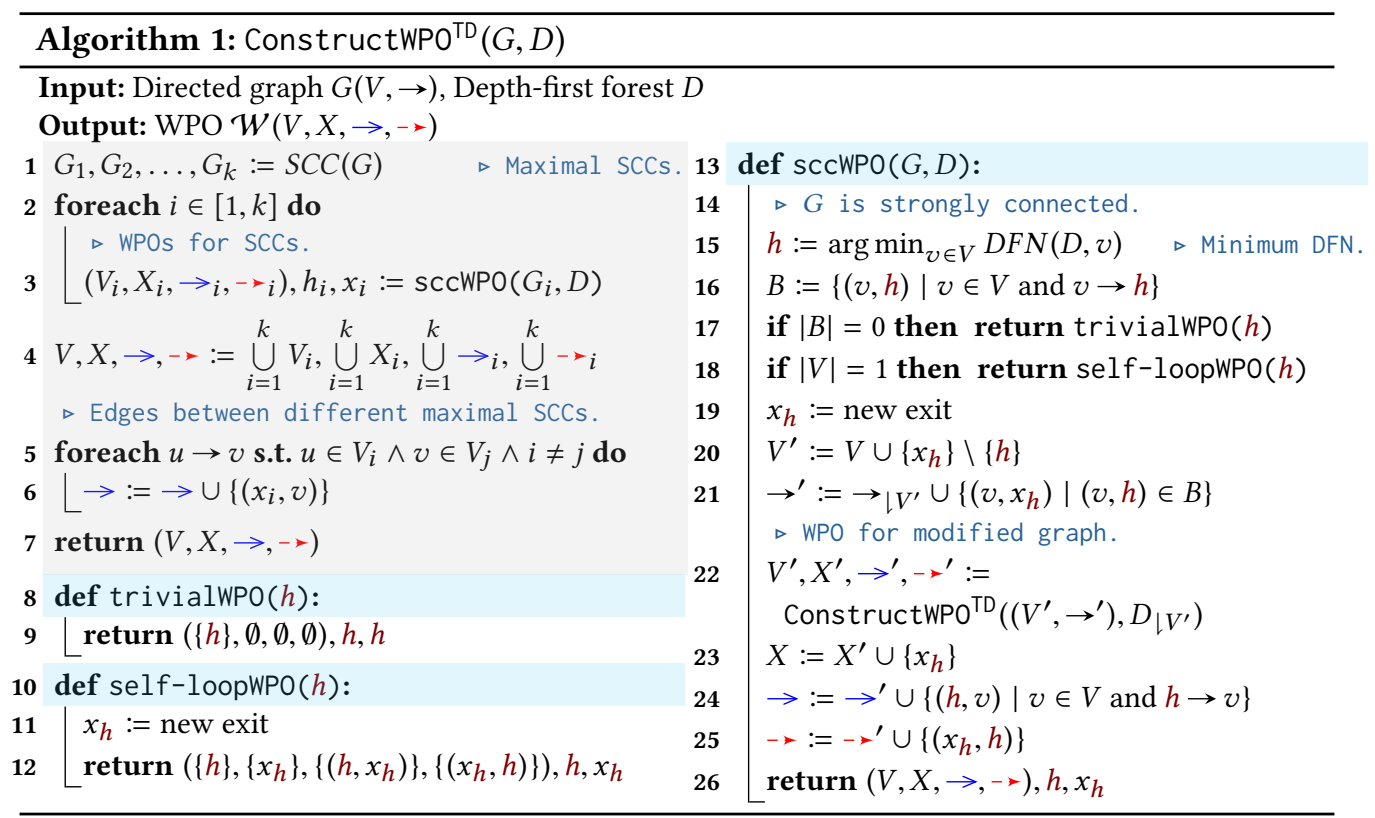

strongly connected components (SCCs) in $G$ on Line 1 . An SCC $G_{i}$ is maximal if there does not exists another SCC that contains all vertices and edges of $G_{i}$. A WPO for an SCC $G_{i}$ is constructed by a call $\operatorname{sccWPO}\left(G_{i}, D\right)$ on Line 3 . This call returns a WPO $\left(V_{i}, X_{i}, \rightarrow_{i},-\rightarrow_{i}\right)$, head $h_{i}$, and exit $x_{i}$. In case of trivial SCCs, the head and the exit are assigned the vertex in $G$. In other cases, the returned value satisfies $\left(x_{i}, h_{i}\right) \in \rightarrow_{i}$ and $\left\lfloor h_{i}, x_{i} \|_{\rightarrow_{i}^{*}}=V_{i} \cup X_{i}\right.$ (Lemma 6.2). Line 4 initializes the WPO for the graph $G$ to union of the WPOs for the SCCs, showing the inductive structure mentioned in Theorem 4.10. On Line 6, scheduling constraints are added for the dependencies that cross the maximal SCCs. $x \rightarrow v$ is added for a dependency $u \rightarrow v$, where $x$ is the exit of the maximal component WPO that contains $u$ but not $v$. This ensures that W5 and $\mathrm{H} 5$ are satisfied.

The function sccWPO takes as input an SCC and its DFF, and returns a WPO, a head, and an exit for this SCC. It constructs the WPO by removing the head $h$ to break the SCC, adding the exit $x_{h}$ as a unique sink, using ConstructWPO ${ }^{\text {TD }}$ to construct a WPO for the modified graph, and appending necessary elements for the removed head. Ignoring the exit $x_{h}$, it shows the inductive structure mentioned in Theorem 4.11. Line 15 chooses a vertex with minimum DFS numbering (DFN) as the head. Incoming edges to the head $h$ are back edges for DFF $D$ on Line 16 because $h$ has the minimum DFN and can reach to all other vertices in the graph. If there are no back edges, then the input SCC is trivial, and a trivial WPO with single element is returned on Line 17. If there is only one vertex in the SCC (with a self-loop), corresponding self-loop WPO is returned on Line 18. For other non-trivial SCCs, $h$ is removed from the graph and newly created $x_{h}$ is added as a unique sink on Lines 19-21. A call ConstructWPO ${ }^{\mathrm{TD}}\left(\left(V^{\prime}, \rightarrow^{\prime}\right), D_{\mid V^{\prime}}\right)$ on Line 22 returns a WPO for the modified graph. Exit $x_{h}$ is moved from $V^{\prime}$ to $X^{\prime}$ on Line 23, scheduling constraints regarding the head $h$ is added on Line 24, and $x_{h} \rightarrow h$ is added on Line 25 to satisfy W5 for the removed back edges.

Example 6.1. Consider the graph $G_{3}$ in Figure 4(a). $\operatorname{SCC}\left(G_{3}\right)$ on Line 1 of ConstructWPO ${ }^{\text {TD }}$ returns a trivial SCC with vertex 1 and three non-trivial SCCs with vertex sets $\{5,6,7,8\},\{2,3\}$, and $\{4\}$. For the trivial SCC, sccWPO on Line 3 returns (1), 1, 1). For the non-trivial SCCs, it 


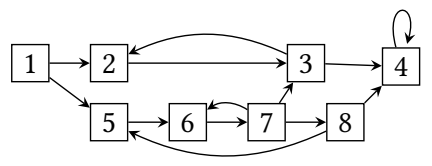

(a)

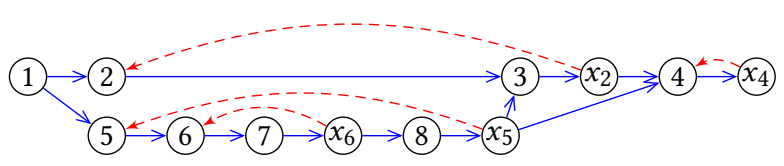

(b)

Fig. 4. (a) Directed graph $G_{3}$ and (b) WPO $\mathcal{W}_{3}$. Vertices $V$ are labeled using DFN; exits $X=\left\{x_{2}, x_{4}, x_{5}, x_{8}\right\}$.

returns $\left(\right.$ (5) $\left.\left.\rightarrow(6) \rightarrow(7) \rightarrow x_{6} \rightarrow(8) \rightarrow x_{5}\right), 5, x_{5}\right),\left(\right.$ (2) $\left.\left.\rightarrow(3) \rightarrow x_{2}\right), 2, x_{2}\right)$, and $\left(\right.$ (4) $\left.\rightarrow\left(x_{4}\right), 4, x_{4}\right)$. On Line 6, scheduling constraints $1 \rightarrow 2,1 \rightarrow 5, x_{5} \rightarrow 3, x_{5} \rightarrow 4$, and $x_{2} \rightarrow 4$ are added for the edges $1 \rightarrow 2$, $1 \rightarrow 5,7 \rightarrow 3,8 \rightarrow 4$, and $3 \rightarrow 4$, respectively. The final result is identical to $\mathcal{W}_{3}$ in Figure $4(\mathrm{~b})$.

Now, consider the execution of sccWPO when given the SCC with vertex set $V=\{5,6,7,8\}$ as input. On Line 15, the vertex 5 is chosen as the head $h$, because it has the minimum DFN among $V$. The set $B$ on Line 16 is $B=\{(8,5)\}$. The SCC is modified on Lines $19-21$, and ConstructWPO ${ }^{\text {TD }}$ on Line 22 returns (6) $\rightarrow(7) \rightarrow x_{6} \rightarrow 8 \rightarrow x_{5}$ for the modified graph. Moving $x_{5}$ from $V^{\prime}$ to $X^{\prime}$ on Line 23, adding $5 \rightarrow 6$ on Line 24, and adding $x_{5} \rightarrow 5$ on Line 25 yields the WPO for the SCC.

Before we prove that the output of ConstructWPO ${ }^{\text {TD }}$ in Algorithm 1 is a WPO, we prove that the output of function sccWPO satisfies the property of a WPO for non-trivial strongly connected graph in Corollary 4.15.

LEMmA 6.2. Given a non-trivial strongly connected graph $G(V, \rightarrow)$ and its depth-first forest $D$, the returned value $(V, X, \rightarrow,-\rightarrow), h, x_{h}$ of $\operatorname{sccWPO}(G, D)$ satisfies $x_{h^{-}} h$ and $\llbracket h, x_{h} \|_{\rightarrow^{*}}=V \cup X$.

Proof. We use structural induction on the input $G$ to prove this. The base case is when $G$ has no non-trivial nested SCCs, and the inductive step uses an induction hypothesis on the non-trivial nested SCCs.

[BASE CASE]: If the graph only has a single vertex $h$ and a self-loop, then $\operatorname{self-loopWPO}(h)$ is returned on Line 18, whose value satisfies the lemma. Otherwise, because there are no non-trivial nested SCCs inside the graph, removing the head $h$ on Line 20 removes all the cycles in the graph. Also, adding a new vertex $x_{h}$ on Line 19 and 21 does not create a cycle, so the modified graph is acyclic. Therefore, $\left(V^{\prime}, \rightarrow^{\prime}\right)$ on Line 22 equals $\left(V^{\prime}, \rightarrow^{\prime}\right)$. Because the input graph is strongly connected, every vertex in the graph is reachable from $h$. This is true even without the back edges because we can ignore the cyclic paths. Also, because the exit $x_{h}$ is a unique sink in the modified graph, $x_{h}$ is reachable from every vertex. It is unique because the negation would imply that there is a vertex in the original graph that has no outgoing edges, contradicting that the input graph is strongly connected. Finally, with changes in Line 24 and 25, we see that the lemma holds for the base case.

[INDUCTIVE STEP]: Let $G_{i}$ be one of the maximal non-trivial nested SCCs that is identified on Line 1. Because $h_{i}$ has the minimum DFN in $G_{i}, h_{i}$ must be an entry of $G_{i}$, and there must exist an incoming scheduling constraints to $h_{i}$. By the induction hypothesis, $u \rightarrow^{+} h_{i}$ implies $u \rightarrow^{+} v$ for all $v \in\left(V_{i} \cup X_{i}\right)$ and $u \notin\left(V_{i} \cup X_{i}\right)$. Also, because scheduling constraints added on Line 6 has exits as their sources, $v \rightarrow^{+} w$ implies $x_{i} \rightarrow^{+} w$ for all $v \in\left(V_{i} \cup X_{i}\right)$ and $w \notin\left(V_{i} \cup X_{i}\right)$. The graph of super-nodes (an SCC contracted to a single vertex) of the modified graph $\left(V^{\prime}, \rightarrow^{\prime}\right)$ is acyclic. Therefore, by applying the similar reasoning as the base case on this graph of super-nodes, we see that the lemma holds.

Armed with the above lemma, we now prove that ConstructWPO ${ }^{\text {TD }}$ constructs a WPO.

Theorem 6.3. Given a graph $G(V, \rightarrow)$ and its depth-first forest $D$, the returned value $(V, X, \rightarrow,-\rightarrow)$ of ConstructWPO ${ }^{\mathrm{TD}}(G, D)$ is a WPO for $G$. 
Proof. We show that the returned value $(V, X, \rightarrow,-\rightarrow)$ satisfies all properties W1-W5 in Definition 4.3.

[W1] $V$ equals the vertex set of the input graph, and $X$ consists only of the newly created exits.

[W2] For all exits, $x_{h} \rightarrow h$ is added on Line 12 and 25 . These are the only places stabilization constraints are created.

[W3] All scheduling constraints are created on Line 6, 12, and 24.

[W4-H1] $\left(\rightarrow_{i}^{*}, V_{i} \cup X_{i}\right)$ is reflexive and transitive by definition. Because the graph with maximal SCCs contracted to single vertices (super-nodes) is acyclic, scheduling constraints on Line 6 cannot create a cycle. Also, Line 24 only adds outgoing scheduling constraints and does not create a cycle. Therefore, $\left(\rightarrow^{*}, V \cup X\right)$ is antisymmetric.

[W4-H2] Exactly one stabilization constraint is created per exit on Line 12 and 25. Because $h$ is removed from the graph afterwards, it does not become a target of another stabilization constraint.

[W4-H3] By Lemma 6.2, $x_{h} \rightarrow h$ implies $h \rightarrow^{+} x_{h}$.

[W4-H4] Because the maximal SCCs on Line 3 are disjoint, by Lemma 6.2, all components $\llbracket h_{i}, x_{i} \rrbracket_{\rightarrow^{*}}$ are disjoint.

[W4-H5] All additional scheduling constraints going outside of a component have exits as their sources on Line 6.

[W5] For $u \rightarrow v$, either (i) scheduling constraint is added in Line 6 and 24, or (ii) stabilization constraint is added in Line 18 and 25. In the case of (i), one can check that the property holds for Line 24 . For Line 6, if $u$ is a trivially maximal SCC, $x_{i}=u$. Else, $u \rightarrow^{*} x_{i}$ by Lemma 6.2, and with added $x_{i} \rightarrow v, u \rightarrow^{+} v$. In the case of (ii), $u \in \llbracket h, x_{h} \rrbracket_{\rightarrow^{*}}$ by Lemma 6.2 where $v=h$.

The next theorem proves that the WPO constructed by ConstructWPO ${ }^{\mathrm{TD}}$ does not include superfluous scheduling constraints, which could reduce parallelism during the fixpoint computation.

TheOREM 6.4. For a graph $G(V, \rightarrow)$ and its depth-first forest $D, W P O W(V, X, \rightarrow,-\rightarrow)$ returned by ConstructWPO ${ }^{\mathrm{TD}}(G, D)$ has the smallest $\rightarrow \rightarrow^{*}$ among the WPOs for $G$ with the same set of $\rightarrow$.

Proof. We use structural induction on the input $G$ to prove this.

[BASE CASE]: The base case is when $G$ is either (i) a trivial SCC or (ii) a single vertex with a self-loop. If $G$ is a trivial SCC, then the algorithm outputs single trivial WPO, whose $\rightarrow$ * is empty.

If $G$ is a single vertex with a self-loop, then there should be at least one exit in the WPO. The algorithm outputs self-loop WPO, whose $\rightarrow^{*}$ contains only $\left(h, x_{h}\right)$.

[INDUCTIVE STEP]: The two cases for the inductive step are (i) $G$ is strongly connected and (ii) $G$ is not strongly connected. If $G$ is strongly connected, then due to Corollary 4.15, all WPOs of $G$ must have a single maximal component that is equal to $V \cup X$. We only consider WPOs with the same set of $\rightarrow$, so $h$, with minimum DFN, is the head of all WPOs that we consider. By the inductive hypothesis, the theorem holds for the returned value of recursive call on Line 22, where the input is the graph without $h$. Because all WPOs have to satisfy $v \rightarrow^{*} x_{h}$ for all $v \in V \cup X$, adding $x_{h}$ does not affect the size of $\rightarrow *$. Also, Line 24 only adds the required scheduling constraints to satisfy W5 for the dependencies whose source is $h$.

If $G$ is not strongly connected, then by the induction hypothesis, the theorem holds for the returned values of self-loopWPO on Line 3 for all maximal SCCs. Line 6 only adds the required scheduling constraints to satisfy $\mathrm{W} 5$ and $\mathrm{H} 5$ for dependencies between different maximal SCCs,

\subsection{Bottom-up Iterative Construction}

Algorithm 2 presents ConstructWPO ${ }^{\mathrm{BU}}(G, D$, lift), an efficient, almost-linear time iterative algorithm for constructing the WPO of a graph $G(V, \rightarrow)$; the role of the boolean parameter lift is explained in $\S 7$, and is assumed to be to be false throughout this section. ConstructWPOBU is also parametrized 


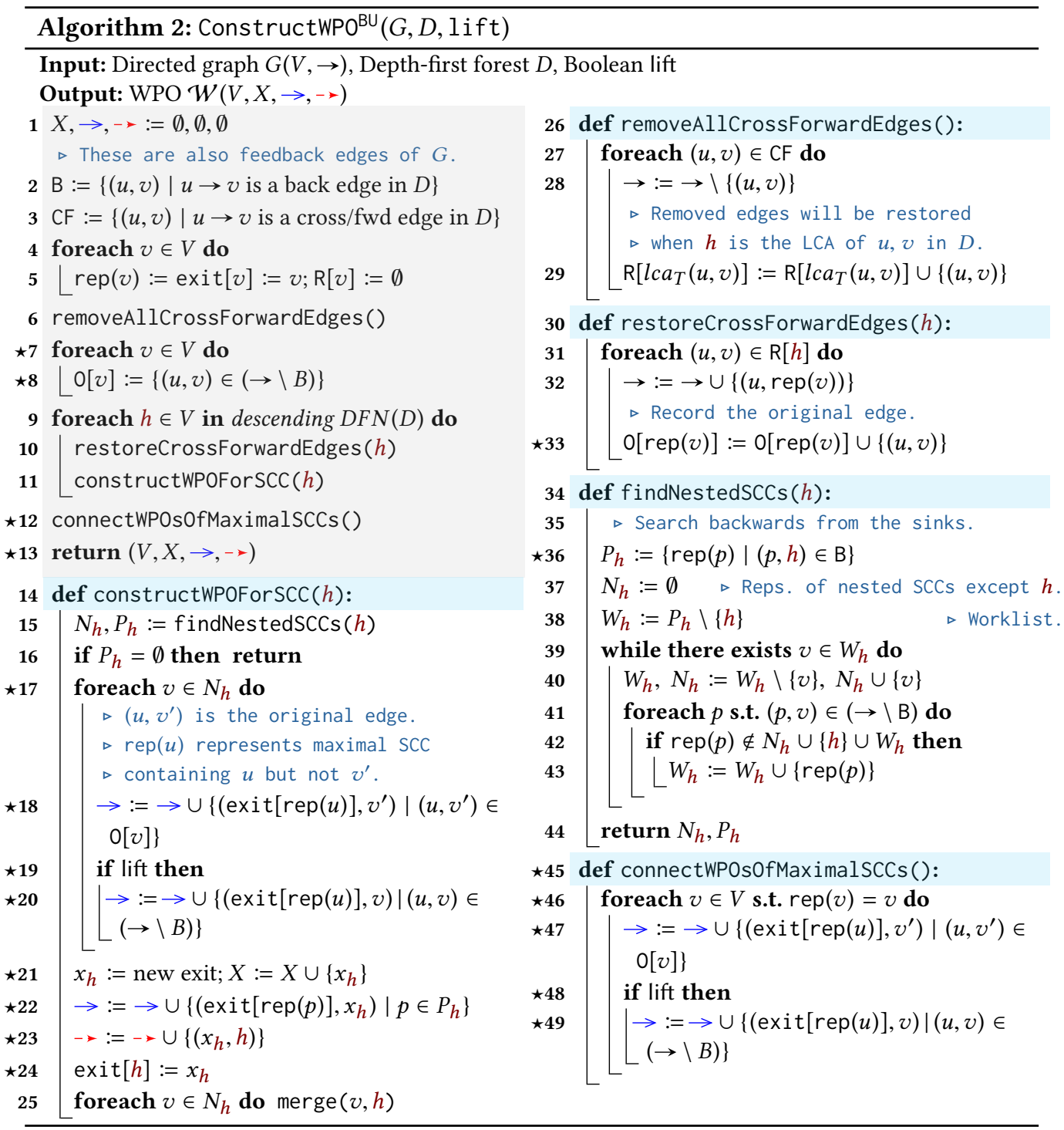

by the depth-first forest $D$ of the graph $G$, and the WPO constructed by the algorithm may change with different forest. DFF defines back edges (B) and cross or forward edges (CF) on Lines 2 and 3.

ConstructWPO ${ }^{\mathrm{BU}}$ maintains a partitioning of the vertices with each subset containing vertices that are currently known to be strongly connected. The algorithm use a disjoint-set data structure to maintain this partitioning. The operation $\operatorname{rep}(v)$ returns the representative of the subset that contains vertex $v$, which is used to determine whether or not two vertices are in the same partition. The algorithm assumes that the vertex with minimum DFN is the representative of the subset. Initially, $\operatorname{rep}(v)=v$ for all vertices $v \in V$. When the algorithm determines that two subsets are strongly connected, they are merged into a single subset. The operation merge $(v, h)$ merges the subsets containing $v$ and $h$, and assigns $h$ to be the representative for the combined subset. 
Example 6.5. Consider the graph $G_{3}$ in Figure 4(a). Let $\underline{1}|\underline{2}| \underline{3}|\underline{4}| \underline{5}|\underline{6} 7| \underline{8}$ be the current partition, where the underline marks the representatives. Thus, $\operatorname{rep}(1)=1$ and $\operatorname{rep}(6)=\operatorname{rep}(7)=6$. If it is found that vertices $5,6,7,8$ are strongly connected, then calls to merge $(6,5)$ and merge $(8,5)$ update the partition to $\underline{1}|\underline{2}| \underline{3}|\underline{4}| \underline{5} 678$. Thus, $\operatorname{rep}(6)=\operatorname{rep}(7)=\operatorname{rep}(8)=\operatorname{rep}(5)=5$.

Auxiliary data structures rep, exit, and R are initialized on Line 5. The map exit maps an SCC (represented by its header $h$ ) to its corresponding exit $x_{h}$. Initially, exit[v] is set to $v$, and updated on Line 24 when a non-trivial SCC is discovered by the algorithm.

The map R maps a vertex to a set of edges, and is used to handle irreducible graphs [Hecht and Ullman 1972; Tarjan 1973]. Initially, $R$ is set to $\emptyset$, and updated on Line 29. The function findNestedSCCs relies on the assumption that the graph is reducible. This function follows the edges backwards to find nested SCCs using $\operatorname{rep}(p)$ instead of predecessor $p$, as on Lines 36 and 43 , to avoid repeatedly searching inside the nested SCCs. $\operatorname{rep}(p)$ is the unique entry to the nested SCC that contains the predecessor $p$ if the graph is reducible. To make this algorithm work for irreducible graphs as well, cross or forward edges are removed from the graph initially by function removeAllCrossForwardEdges (called on Line 6) to make the graph reducible. Removed edges are then restored by function restoreCrossForwardEdges (called on Line 10) right before the edges are used. The graph is guaranteed to be reducible when restoring a removed edge $u \rightarrow v$ as $u \rightarrow \operatorname{rep}(v)$ when $h$ is the lowest common ancestor (LCA) of $u, v$ in the depth-first forest. Cross and forward edges are removed on Line 28 and are stored at their LCAs in $D$ on Line 29. Then, as $h$ hits the LCAs in the loop, the removed edges are restored on Line 32. Because the graph edges are modified, map 0 is used to track the original edges. $0[v]$ returns set of original non-back edges that now targets $v$ after the modification. The map 0 is initialized on Line 8 and updated on Line 33.

The call to function constructWPOForScC $(h)$ on Line 11 constructs a WPO for the largest SCC such that $h=\arg \min _{v \in V^{\prime}} \operatorname{DFN}(D, v)$, where $V^{\prime}$ is the vertex set of the SCC. For example, constructWPOForSCC(5) constructs WPO for SCC with vertex set $\{5,6,7,8\}$. Because the loop on Line 9 traverses the vertices in descending DFN, the WPO for a nested SCC is constructed before that of the enclosing SCC. For example, constructWPOForSCC(6) and constructWPOForSCC(8) are called before constructWPOForSCC(5), which construct WPOs for nested SCCs with vertex sets $\{6,7\}$ and $\{8\}$, respectively. Therefore, constructWPOForSCC $(h)$ reuses the constructed sub-WPOs.

The call to function findNestedSCCs $(h)$ on Line 15 returns $N_{h}$, the representatives of the nested SCCs $N_{h}$, as well as $P_{h}$, the predecessors of $h$ along back edges. If $P_{h}$ is empty, then the SCC is trivial, and the function immediately returns on Line 16. Line 18 adds scheduling constraints for the dependencies crossing the nested SCCs. As in ConstructWPO'D , this must be from the exit of maximal SCC that contains $u$ but not $v^{\prime}$ for $u \rightarrow v^{\prime}$. Because $u \rightarrow v^{\prime}$ is now $u \rightarrow \operatorname{rep}\left(v^{\prime}\right), 0[v]$, where $v=\operatorname{rep}\left(v^{\prime}\right)$, is looked up to find $u \rightarrow v^{\prime}$. exit is used to find the exit, where $\operatorname{rep}(u)$ is the representative of maximal SCC that contains $u$ but not $v$. If the parameter lift is true, then scheduling constraint targeting $v$ is also added, forcing all scheduling predecessors outside of a component to be visited before the component's head. Similarly, function connectWPOsOfMaximalSCCs is called after the loop on Line 47 to connect WPOs of maximal SCCs. The exit, scheduling constraints to the exit, and stabilization constraints are added on Lines 21-23. After the WPO is constructed, Line 24 updates the map exit, and Line 25 updates the partition.

Example 6.6. Table 1 describes the steps of ConstructWPO ${ }^{\mathrm{BU}}$ for the irreducible graph $G_{3}$ (Figure 4 ). The $\rightarrow$ updates column shows the modifications to the graph edges, the Current partition column shows the changes in the disjoint-set data structure, and the Current WPO column shows the WPO constructed so far. Each row shows the changes made in each step of the algorithm. Row 'Init' shows the initialization step on Lines $5-6$. Row ' $h=k$ ' shows the $k$-th iteration of the loop 
Table 1. Steps of ConstructWPOBU for graph $G-3$ in Figure 4.

\begin{tabular}{|c|c|c|c|}
\hline Step & $\rightarrow$ updates & Current partition & Current WPO \\
\hline Init & $\begin{array}{l}\{(7,3),(8,4)\} \\
\quad \text { removed }\end{array}$ & $\underline{1}|\underline{2}| \underline{3}|\underline{4}| \underline{5}|\underline{6}| \underline{7} \mid \underline{8}$ & $\begin{array}{l}\text { (1) (2) } \\
\text { (5) (6) } 7 \text { (3) }\end{array}$ \\
\hline$h=8$ & - & $\underline{1}|\underline{2}| \underline{3}|\underline{4}| \underline{5}|\underline{6}| \underline{7} \mid \underline{8}$ & $\begin{array}{l}\text { (1) }(2) \\
\text { (5) (6) (7) } 8\end{array}$ \\
\hline$h=7$ & - & $\underline{1}|\underline{2}| \underline{3}|\underline{4}| \underline{5}|\underline{6}| \underline{7} \mid \underline{8}$ & $\begin{array}{l}\text { (1) (2) } \\
\text { (5) (6) (7) } 8 \text { (4) }\end{array}$ \\
\hline$h=6$ & - & $\underline{1}|\underline{2}| \underline{3}|\underline{4}| \underline{5}|\underline{6} 7| \underline{8}$ & $\begin{array}{l}\text { (1) (2) } \\
\text { (5) (6) }>\text { (7) }>\left(x_{6}\right)\end{array}$ \\
\hline$h=5$ & - & $\underline{1}|\underline{2}| \underline{3}|\underline{4}| \underline{5} 678$ & (3) \\
\hline$h=4$ & - & $\underline{1}|\underline{2}| \underline{3}|\underline{4}| \underline{5} 678$ & (3) $(4)>x_{4}$ \\
\hline$h=3$ & - & $\underline{1}|\underline{2}| \underline{3}|\underline{4}| \underline{5} 678$ & $\Rightarrow\left(x_{6} \rightarrow(8) \rightarrow x_{5}\right.$ \\
\hline$h=2$ & - & $\underline{1}|\underline{2} 3| \underline{4} \mid \underline{5} 678$ & $\begin{array}{l}\rightarrow(3) \rightarrow\left(x_{2}\right)(4) \rightarrow x_{4} \\
\left.x_{5}\right)\end{array}$ \\
\hline$h=1$ & $\begin{array}{l}\{(7,2),(8,4)\} \\
\quad \text { added }\end{array}$ & $\underline{1}|\underline{2} 3| \underline{4} \mid \underline{5} 678$ & $\begin{array}{l}x_{6}>(3)>\left(x_{2}\right)(4)>x_{4} \\
x_{6} \rightarrow x_{5}\end{array}$ \\
\hline Final & - & $\underline{1}|\underline{2} 3| \underline{4} \mid \underline{5} 678$ & \\
\hline
\end{tabular}

on Lines 9-11. The loop iterates over the vertices in descending DFN: 8, 7, 6, 5, 4, 3, 2, 1. Row 'Final' shows the final step after the loop on Line 12.

During initialization, the cross or forward edges $\{(7,3),(8,4)\}$ are removed, making $G_{3}$ reducible. These edges are added back as $\{(7, \operatorname{rep}(3)),(8, \operatorname{rep}(4))\}=\{(7,2),(8,4)\}$ in $h=1$, where 1 is the LCA of both $(7,3)$ and $(8,4) . G_{3}$ remains reducible after restoration. In step $h=5$, WPOs for the nested SCCs are connected with $5 \rightarrow 6$ and $x_{6} \rightarrow 8$. The new exit $x_{5}$ is created, connected to the WPO via 
$8 \rightarrow x_{5}$, and $x_{5} \rightarrow 5$ is added. Finally, $1 \rightarrow 2,1 \rightarrow 5, x_{5} \rightarrow 3, x_{5} \rightarrow 4$, and $x_{2} \rightarrow 4$ are added, connecting the WPOs for maximal SCCs. If lift is true, then scheduling constraint $x_{5} \rightarrow 2$ is added.

ConstructWPO ${ }^{\mathrm{BU}}$ adapts Tarjan-Havlak-Ramaligam's almost-linear time algorithm [Ramalingam 1999] for constructing Havlak's loop nesting forest (LNF) [Havlak 1997]. Similar to components in the WPO, an LNF represents the nesting relationship among the SCCs. A WPO contains additional information in the form of scheduling constraints, which are used to generate the concurrent iteration strategy for fixpoint computation. Lines in ConstructWPO ${ }^{\mathrm{BU}}$ indicated by $\star$ were added to the algorithm in Ramalingam [1999]. The following two theorems prove the correctness and runtime efficiency of ConstructWPO ${ }^{\mathrm{BU}}$.

Theorem 6.7. Given a graph $G(V, \rightarrow)$ and its depth-first forest $D$, ConstructWPO ${ }^{\mathrm{TD}}(G, D)$ and ConstructWPO ${ }^{\mathrm{BU}}(G, D$, false) construct the same WPO for $G$.

Proof. Using the constructive characterization of an LNF [Ramalingam 2002, Definition 3], an LNF of a graph is constructed by identifying the maximal SCCs, choosing headers for the maximal SCCs, removing incoming back edges to the headers to break the maximal SCCs, and repeating this on the subgraphs. In particular, Havlak's LNF is obtained when the vertex with minimum DFN in the SCC is chosen as the header [Ramalingam 2002, Definition 6]. This construction is similar to how the maximal SCCs are identified on Line 1 of ConstructWPO ${ }^{\mathrm{TD}}$ and how ConstructWPO ${ }^{\mathrm{TD}}$ is called recursively on Line 22. Because ConstructWPO ${ }^{\mathrm{BU}}$ is based on the LNF construction algorithm, both algorithms identify the same set of SCCs, resulting in the same $X$ and $\rightarrow$.

Now consider $u \rightarrow v^{\prime}$ in the original graph that is not a back edge. Let $v$ be $\operatorname{rep}\left(v^{\prime}\right)$ when restoring the edge if the edge is cross/forward, or $v^{\prime}$ otherwise. If both $u, v^{\prime}$ are in some non-trivial SCC, then $v \in N_{h}$ on Line 17 for some $h$. In this case, $\operatorname{rep}(u)$ must be in $N_{h}$. If not, $u \rightarrow v$ creates an entry to the SCC other than $h$. We know that $h$ must be the entry because it has minimum DFN. This contradicts that the modified graph is reducible over the whole run [Ramalingam 1999, Section 5] Because all nested SCCs have been already identified, $\operatorname{rep}(u)$ is the representative of the maximal SCC that contains $u$ but not $v$. Also, if no SCC contains both $u, v^{\prime}$, then $v=\operatorname{rep}(v)$ on Line 46 Because all maximal SCCs are found, $\operatorname{rep}(u)$ returns the representative of the maximal SCC that contains $u$ but not $v$. Therefore, Line 18 and Line 47 construct the same $\rightarrow$ as ConstructWPO ${ }^{\text {TD }}$.

Theorem 6.8. The running time of ConstructWPO ${ }^{\mathrm{BU}}(G, D, *)$ is almost-linear.

Proof. The non-starred lines in ConstructWPOBU are the same as Tarjan-Havlak-Ramalingam's almost-linear time algorithm for constructing Havlak's LNF [Ramalingam 1999, Section 5]. The starred lines only add constant factors to the algorithm. Thus, the running time of ConstructWPO ${ }^{\mathrm{BU}}$ is also almost-linear.

\section{CONNECTION TO WEAK TOPOLOGICAL ORDER}

The weak partial order defined in $\S 4$ is a strict generalization of weak topological order (WTO) defined by Bourdoncle [1993]. Let us first recall the definitions by Bourdoncle.

Definition 7.1. A hierarchical total order (HTO) of a set $S$ is a well-parenthesized permutation of this set without two consecutive "(".

An HTO is a string over the alphabet $S$ augmented with left and right parenthesis. An HTO of $S$ induces a total order $\leq$ over the elements of $S$. The elements between two matching parentheses are called a component, and the first element of a component is called the head. The set of heads of the components containing the element $l$ is denoted by $\omega(l)$.

Definition 7.2. A weak topological order (WTO) of a directed graph is a HTO of its vertices such that for every edge $u \rightarrow v$, either $u<v$ or $v \leq u$ and $v \in \omega(u)$. 


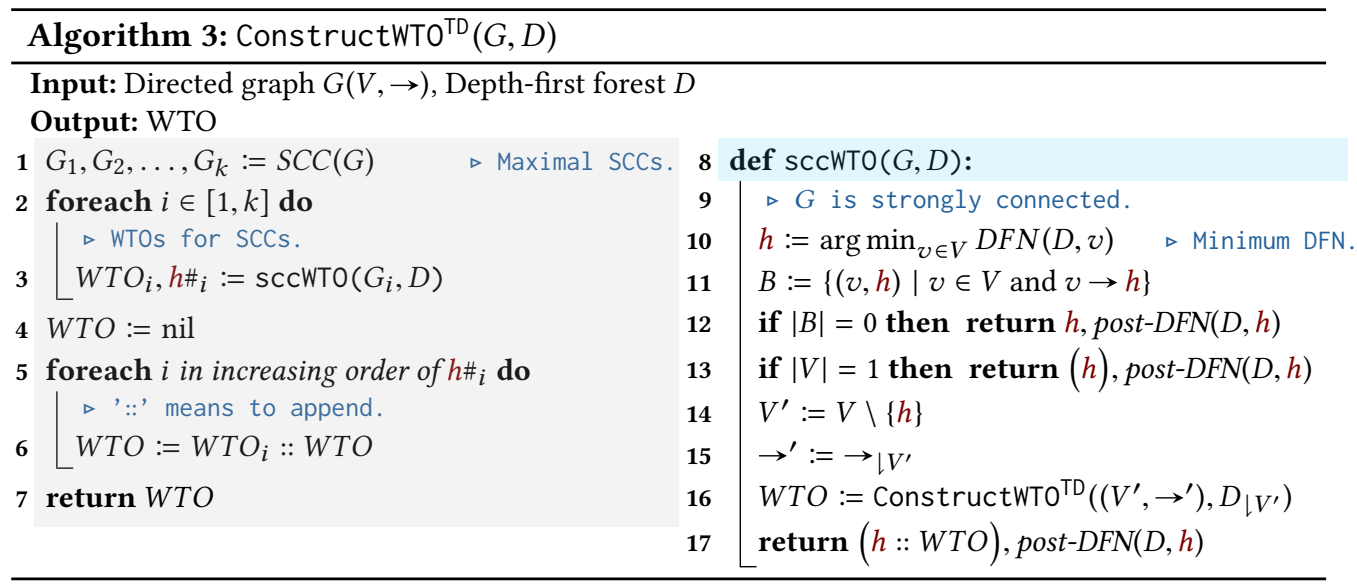

A WTO factors out a feedback edge set from the graph using the matching parentheses and topologically sorts the rest of the graph to obtain a total order of vertices. A feedback edge set defined by a WTO is $\{(u, v) \in \rightarrow \mid v \leq u$ and $v \in \omega(u)\}$.

Example 7.3. The WTO of graph $G_{1}$ in Figure 2(a) is 1 (2 (3 4) (6 79 8) 5) 10. The feedback edge set defined by this WTO is $\{(4,3),(8,6),(5,2)\}$, which is the same as that defined by WPO $\mathcal{W}_{1}$.

Algorithm 3 presents a top-down recursive algorithm for constructing a WTO for a graph $G$ and its depth-first forest $D$. Notice the use of increasing post DFN order when merging the results on Line 5. In general, a reverse post DFN order of a graph is its topological order. Therefore, ConstructWTO ${ }^{\mathrm{TD}}$, in effect, topologically sorts the DAG of SCCs recursively. Because it is recursive, it preserves the components and their nesting relationship. Furthermore, by observing the correspondence between ConstructWTO ${ }^{\text {TD }}$ and ConstructWPO ${ }^{\text {TD }}$, we see that ConstructWTO ${ }^{\mathrm{TD}}(G, D)$ and ConstructWPO ${ }^{\mathrm{TD}}(G, D, *)$ construct the same components with same heads and nesting relationship.

The definition of HPO (Definition 4.1) generalizes the definition of HTO (Definition 7.1) to partial orders, while the definition of WPO (Definition 4.3) generalizes the definition of WTO (Definition 7.2) to partial orders. In other words, the two definitions define the same structure if we strengthen $\mathrm{H} 1$ to a total order. If we view the exits in $X$ as closing parenthesis ")" and $x \rightarrow h$ as matching parentheses $(h \ldots)$, then the correspondence between the two definitions becomes clear. The conditions that a HTO must be well-parenthesized and that it disallows two consecutive "(" correspond to conditions $\mathrm{H} 4, \mathrm{H} 2$, and W1. While $\mathrm{H} 5$ is not specified in Bourdoncle's definition, it directly follows from the fact that $\leq$ is a total order. Finally, the condition in the definition of WTO matches W5. Thus, using the notion of WPO, we can define a WTO as:

Definition 7.4. A weak topological order (WTO) for a graph $G(V, \rightarrow)$ is a WPO $\mathcal{W}(V, X, \rightarrow,-\rightarrow)$ for $G$ where $\left(V \cup X, \rightarrow^{*}\right)$ is a total order.

Definition 7.4 hints at how a WTO for a graph can be constructed from a WPO. The key is to construct a linear extension of the partial order $\left(V \cup X, \rightarrow^{*}\right)$ of the WPO, while ensuring that properties $\mathrm{H} 1, \mathrm{H} 4$, and $\mathrm{H} 5$ continue to hold. ConstructWTO ${ }^{\mathrm{BU}}$ (Algorithm 4) uses the above insight to construct a WTO of $G$ in almost-linear time, as proved by the following two theorems.

THEOREM 7.5. Given a directed graph $G$ and its depth-first forest $D$, the returned value $(V, X, \rightarrow,-\rightarrow)$ of ConstructWTO ${ }^{B U}(G, D)$ is a WTO for $G$.

Proof. The call ConstructWPO ${ }^{\mathrm{BU}}(G, D$, true $)$ on Line 1 constructs a WPO for $G$. With lift set to true in ConstructWPO ${ }^{\mathrm{BU}}$, all scheduling predecessors outside of a component are visited before 


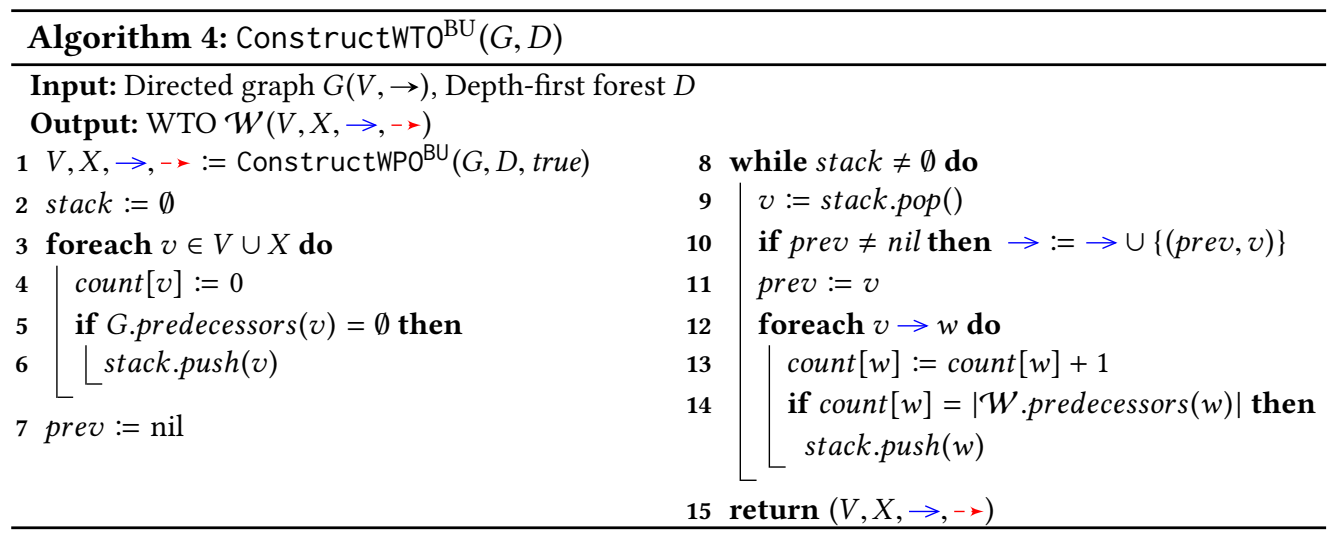

the head of the component. The algorithm then visits the vertices in topological order according to $\rightarrow$. Thus, the additions to $\rightarrow$ on Line 10 do not violate $\mathrm{H} 1$ and lead to a total order $\left(V \cup X, \rightarrow^{*}\right)$. Furthermore, because a stack is used as the worklist and because of $\mathrm{H} 5$, once a head of a component is visited, no element outside the component is visited until all elements in the component are visited. Therefore, the additions to $\rightarrow$ on Line 10 preserve the components and their nesting relationship, satisfying H4. Because the exit is the last element visited in the component, no scheduling constraint is added from inside of the component to outside, satisfying H5. Thus, ConstructWTo ${ }^{\mathrm{BU}}(G, D)$ constructs a WTO for $G$.

Example 7.6. For graph $G_{1}$ in Figure 2(a), ConstructWTO ${ }^{\mathrm{BU}}\left(G_{1}, D\right)$ returns the following WTO:

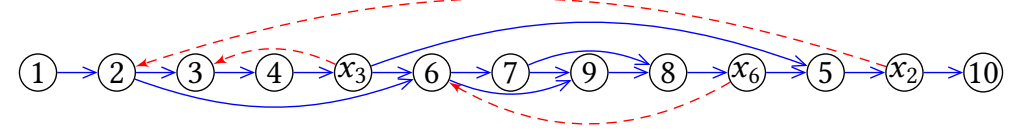

ConstructWTO ${ }^{\mathrm{BU}}$ first constructs the WPO $\mathcal{W}_{1}$ in Figure $2(\mathrm{~b})$ using ConstructWPO ${ }^{\mathrm{BU}}$. The partial order $\rightarrow^{*}$ of $\mathcal{W}_{1}$ is extended to a total order by adding $x_{3} \rightarrow 6$ and $7 \rightarrow 9$. The components and their nesting relationship in $\mathcal{W}_{1}$ are preserved in the constructed WTO. This WTO is equivalent to 1 (2 (3 4) (6 79 8) 5) 10 in Bourdoncle's representation (see Definition 7.2 and 7.3).

Theorem 7.7. Running time of ConstructWTOBU $(G, D)$ is almost-linear.

Proof. The call to ConstructWPO ${ }^{\mathrm{BU}}$ in ConstructWTO ${ }^{\mathrm{BU}}$ takes almost-linear time, by Theorem 6.8. After this call the elements and scheduling constraints are visited only once. Thus, the running time of ConstructWTO ${ }^{\mathrm{BU}}$ is almost-linear.

Bourdoncle [1993] presents a more efficient version of the ConstructWTO ${ }^{\text {TD }}$ for WTO construction; however, it has worst-case cubic time complexity. Thus, Theorem 7.7 improves upon the previously known algorithm for WTO construction.

The next theorem shows that ConstructWTO ${ }^{\mathrm{BU}}$ outputs the same WTO as ConstructWTO ${ }^{\text {TD }}$.

Theorem 7.8. Given a directed graph $G$ and its depth-first forest $D$, ConstructWTO ${ }^{B U}(G, D)$ and ConstructWTO ${ }^{\mathrm{TD}}(G, D)$ construct the same WTO for $G$.

Proof. We shown above, ConstructWTO ${ }^{\mathrm{TD}}(G, D)$ and ConstructWPO ${ }^{\mathrm{TD}}(G, D)$ construct the same components with same heads and nesting relationship. Therefore, using Theorem 6.7, we can conclude that ConstructWTOBU $(G, D)$ and ConstructWTO ${ }^{\mathrm{TD}}(G, D)$ construct the same WTO. 
The next theorem shows that our concurrent fixpoint algorithm in Figure 3 computes the same fixpoint as Bourdoncle's sequential fixpoint algorithm.

THEOREM 7.9. The fixpoint computed by the concurrent fixpoint algorithm in Figure 3 using the WPO constructed by Algorithm 2 is the same as the one computed by the sequential Bourdoncle's algorithm that uses the recursive iteration strategy.

Proof. With both stabilization constraint, $-\rightarrow$, and matching parentheses, (...), interpreted as the "iteration until stabilization" operator, our concurrent iteration strategy for ConstructWTO ${ }^{\mathrm{BU}}(G, D)$ computes the same fixpoint as Bourdoncle's recursive iteration strategy for ConstructWTO ${ }^{\text {TD }}(G, D)$. The only change we make to a WPO in ConstructWTO ${ }^{\mathrm{BU}}$ is adding more scheduling constraints. Further, Theorem 5.6 proved that our concurrent iteration strategy is deterministic. Thus, our concurrent iteration strategy computes the same fixpoint when using the WPO constructed by either ConstructWTO $\mathrm{BU}^{\mathrm{BU}}(G, D)$ or ConstructWPO $\mathrm{BU}(G, D$, false). Therefore, our concurrent fixpoint algorithm in Figure 3 computes the same fixpoint as Bourdoncle's sequential fixpoint algorithm.

\section{IMPLEMENTATION}

Our deterministic parallel abstract interpreter, which we called PIKOs, was built using IKOS [Brat et al. 2014], an abstract-interpretation framework for C/C++ based on LLVM.

Sequential baseline IKOS. IKOS performs interprocedural analysis to compute invariants for all programs points, and can detect and prove the absence of runtime errors in programs. To compute the fixpoint for a function, IKOS constructs the WTO of the CFG of the function and uses Bourdoncle's recursive iteration strategy [Bourdoncle 1993]. Context sensitivity during interprocedural analysis is achieved by performing dynamic inlining during fixpoint: formal and actual parameters are matched, the callee is analyzed, and the return value at the call site is updated after the callee returns. This inlining also supports function pointers by resolving the set of possible callees and joining the results.

PIкos. We modified IKOS to implement our deterministic parallel abstract interpreter using Intel's Threading Building Blocks (TBB) library [Reinders 2007]. We implemented the almost-linear time algorithm for WPO construction (§6). We implemented the deterministic parallel fixpoint iterator (§5) using TBB’s parallel_do. Multiple callees at an indirect call site are analyzed in parallel using TBB's parallel_reduce. We refer to this extension of IKOS as PIKOs; we use PIKos $\langle k\rangle$ to refer to the instantiation of Pikos that uses up to $k$ threads.

Path-based task spawning in PIkos. PIxos relies on TBB's tasks to implement the parallel fixpoint iterator. Our initial implementation would spawn a task for each WPO element when it is ready to be scheduled. Such a naive approach resulted PIKOs being slower than IKOS; there were 10 benchmarks where speedup of PIKOS $\langle 2\rangle$ was below 0.90x compared to IKOS, with a minimum speedup of $0.74 \mathrm{x}$. To counter such behavior, we implemented a simple path-based heuristic for spawning tasks during fixpoint computation. We assign ids to each element in the WPO $W$ as follows: assign id 1 to the elements along the longest path in $W$, remove these elements from $W$ and assign id 2 to the elements along the longest path in the resulting graph, and so on. The length of the path is based on the number of instructions as well as the size of the functions called along the path. During the fixpoint computation, a new task is spawned only if the id of the current element differs from that of the successor that is ready to be scheduled. Consequently, elements along critical paths are executed in the same task. 
Memory allocator for concurrency. We experimented with three memory allocators optimized for parallelism: Tcmalloc, ${ }^{3}$ Jemalloc, ${ }^{4}$ and Tbbmalloc [Reinders 2007]. Tcmalloc was chosen because it performed the best in our settings for both PIKOs and IKOS.

Abstract domain. Our fixpoint computation algorithm is orthogonal to the abstract domain in use. PIKOS $\langle k\rangle$ works for all abstract domains provided by IKOS as long as the domain was thread-safe. These abstract domains include interval [Cousot and Cousot 1977], congruence [Granger 1989], gauge [Venet 2012], and DBM [Miné 2001]. Variable-packing domains [Gange et al. 2016] could not be used because their implementations were not thread-safe. We intend to explore thread-safe implementations for these domains in the future.

\section{EXPERIMENTAL EVALUATION}

In this section, we study the runtime performance of Pıкоs (§8) on a large set of C programs using IKOS as the baseline. The experiments were designed to answer the following questions:

RQ0 [Determinism] Is PIKos deterministic? Is the fixpoint computed by PIKos the same as that computed by IKOS?

RQ1 [Performance] How does the performance of PIKOS $\langle 4\rangle$ compare to that of IKOS?

RQ2 [Scalability] How does the performance of Pikos $\langle k\rangle$ scale as we increase the number of threads $k$ ?

Platform. All experiments were run on Amazon EC2 C5, which use $3.00 \mathrm{GHz}$ Intel Xeon Platinum 8124M CPUs. IKOS and Pikos $\langle k\rangle$ with $1 \leq k \leq 4$ were run on c5.2xlarge (8 vCPUs, 4 physical cores, 16GB memory), PIKos $\langle k\rangle$ with $5 \leq k \leq 8$ on c5.4xlarge (16 vCPUs, 8 physical cores, 32GB memory), and Pikos $\langle k\rangle$ with $9 \leq k$ on c5.9xlarge (36 vCPUs, 18 physical cores, 72GB memory). Dedicated EC2 instances and BenchExec [Beyer et al. 2019] were used to improve reliability of timing results. The Linux kernel version was 4.4, and gcc 8.1.0 was used to compile PIKOs $\langle k\rangle$ and IKOS.

Abstract Domain. We experimented with both interval and gauge domain, and the analysis precision was set to track immediate values, pointers, and memory. The results were similar for both interval and gauge domain. We show the results using the interval domain. Because we are only concerned with the time taken to perform fixpoint computation, we disabled program checks, such as buffer-overflow detection, in both IKOS and PIKOs.

Benchmarks. We chose 4319 benchmarks from the following two sources:

SVC We selected all 2701 benchmarks from the Linux, control-flows, and loops categories of SV-COMP 2019 [Beyer 2019]. These categories are well suited for numerical analysis, and have been used in recent work [Singh et al. 2018a,b]. Programs from these categories have indirect function calls with multiple callees at a single call site, large switch statements, nested loops, and irreducible CFGs.

OSS We selected all 1618 programs from the Arch Linux core packages that are primarily written in $\mathrm{C}$ and whose LLVM bitcode are obtainable by gllvm. ${ }^{5}$ These include, but are not limited to, apache, coreutils, cscope, curl, dhcp, fvwm, gawk, genius, ghostscript, gnupg, iproute, ncurses, nmap, openssh, postfix, $r$, socat, vim, wget, etc.

We checked that the time taken by IKOS and PIKOS $\langle 1\rangle$ was the same; thus, any speedup achieved by PIKos $\langle k\rangle$ is due to parallelism in the fixpoint computation. Note that the time taken for WTO and WPO construction is very small compared to actual fixpoint computation, which is why PIKOS $\langle 1\rangle$ does not outperform IKOS. The almost-linear algorithm for WPO construction (§ 6.2)

\footnotetext{
${ }^{3}$ https://gperftools.github.io/gperftools/tcmalloc.html

${ }^{4}$ http://jemalloc.net/

${ }^{5}$ https://github.com/SRI-CSL/gllvm
} 


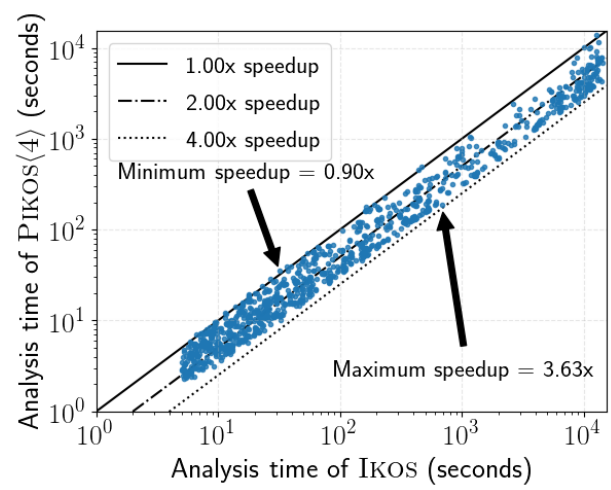

Fig. 5. Log-log scatter plot of analysis time taken by IKOS and PIKоs $\langle 4\rangle$ on 1017 benchmarks. Speedup is defined as the analysis time of IKOS divided by analysis time of PIKOS〈4〉. 1.00x, 2.00x, and 4.00x speedup lines are shown. Benchmarks that took longer to analyze in IKOS tended to have higher speedup.

Table 2. A sample of the 1017 results in Figure 5. The first 5 rows list benchmarks with the highest speedup, and the remaining 5 rows list benchmarks with the longest analysis time in IKOS.

\begin{tabular}{lllll}
\hline Benchmark & Src. & IKOS (s) & PIKOS $\langle 4\rangle(\mathrm{s})$ & Speedup \\
\hline audit-2.8.4/aureport & OSS & 684.29 & 188.25 & $3.63 \mathrm{x}$ \\
feh-3.1.3/feh & OSS & 9004.83 & 2534.91 & $3.55 \mathrm{x}$ \\
ldv-linux-4.2-rc1/43_2a-crypto & SVC & 10051.39 & 2970.02 & $3.38 \mathrm{x}$ \\
ratpoison-1.4.9/ratpoison & OSS & 1303.73 & 387.70 & $3.36 \mathrm{x}$ \\
ldv-linux-4.2-rc1/08_1a-gpu-amd & SVC & 2002.80 & 602.06 & $3.33 \mathrm{x}$ \\
\hline fvwm-2.6.8/FvwmForm & OSS & 14368.70 & 6913.47 & $2.08 \mathrm{x}$ \\
ldv-linux-4.2-rc1/32_7a-ata & SVC & 14138.04 & 7874.58 & $1.80 \mathrm{x}$ \\
ldv-linux-4.2-rc1/43_2a-ata & SVC & 14048.39 & 7925.82 & $1.77 \mathrm{x}$ \\
ldv-linux-4.2-rc1/43_2a-scsi-mpt3sas & SVC & 14035.69 & 4322.59 & $3.25 \mathrm{x}$ \\
ldv-linux-4.2-rc1/08_1a-staging-rts5208 & SVC & 13540.72 & 7147.69 & $1.89 \mathrm{x}$ \\
\hline
\end{tabular}

is an interesting theoretical result, which shows a new connection between the algorithms of Bourdoncle and Ramalingam. However, the practical impact of the new algorithm is in preventing stack overflow in the analyzer that occurs when using a recursive implementation of Bourdoncle's WTO construction algorithm; see the GitHub issues linked in the footnotes in $\S 1$.

There were 130 benchmarks for which IKOS took longer than 4 hours. To include these benchmarks, we made the following modification to the dynamic function inliner, which implements the context sensitivity in interprocedural analysis in both IKOS and PIKos: if the call depth during the dynamic inlining exceeds the given limit, the analysis returns $T$ for that callee. For each of the 130 benchmarks, we determined the largest limit for which IKOS terminated within 4 hours. Because our experiments are designed to understand the performance improvement in fixpoint computation, we felt this was a reasonable thing to do.

\subsection{RQ0: Determinism of PIкоs}

As a sanity check for our theoretical results, we experimentally validated Theorem 5.6 by running Pikos multiple times with varying number of threads, and checked that the final fixpoint was always the same. Furthermore, we experimentally validated Theorem 7.9 by comparing the fixpoints computed by Pikos and IKOS. 

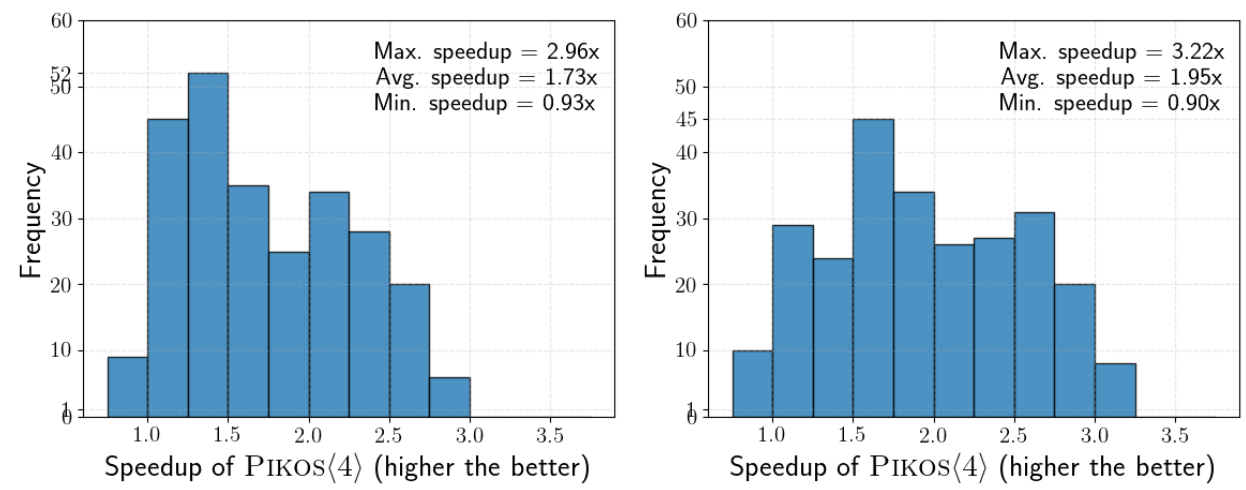

(a) $0 \% \sim 25 \%$ (5.02 seconds 16.01 seconds)

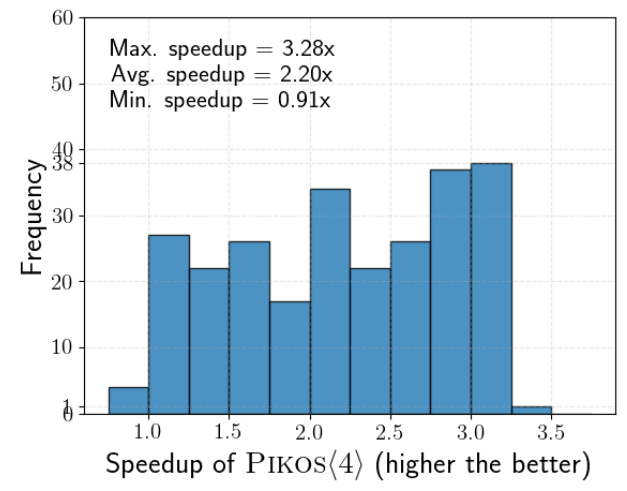

(b) $25 \% \sim 50 \%$ (16.04 seconds 60.45 seconds)

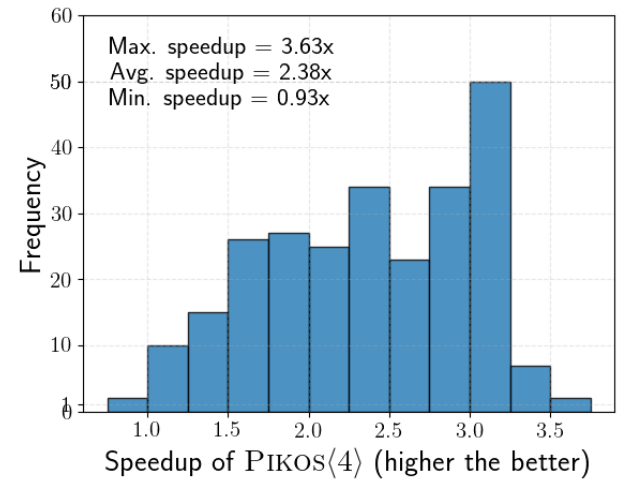

(c) $50 \% \sim 75 \%$ (60.85 seconds 508.14 seconds)

(d) $75 \% \sim 100 \%$ ( 508.50 seconds 14368.70 seconds)

Fig. 6. Histograms of speedup of PIкоS $\langle 4\rangle$ for different ranges. Figure 6(a) shows the distribution of benchmarks that took from 5.02 seconds to 16.01 seconds in IKOS. They are the bottom $25 \%$ in terms of the analysis time in IKOS. The distribution tended toward a higher speedup in the upper range.

\subsection{RQ1: Performance of PIKos $\langle 4\rangle$ compared to IKOS}

We exclude results for benchmarks for which IKOS took less than 5 seconds. For these benchmarks, the average analysis time of IKOS was 0.76 seconds. The analysis time of IKOS minus the analysis time of PIKos $\langle 4\rangle$ ranged from +2.81 seconds (speedup in PIKos $\langle 4\rangle$ ) to -0.61 seconds (slowdown in Pikos $\langle 4\rangle$ ), with an average of +0.16 seconds. Excluding these benchmarks left us with 1017 benchmarks, consisting of 518 SVC and 499 OSS benchmarks.

Figure 5 shows a log-log scatter plot, comparing the analysis time of Piкоs $\langle 4\rangle$ with that of IKOS for each of the 1017 benchmarks. Speedup is defined as the analysis time of IKOS divided by the analysis time of PIKos $\langle 4\rangle$. The maximum speedup was $3.63 \mathrm{x}$, which is close to the maximum speedup of 4.00x. Arithmetic, geometric, and harmonic mean of the speedup were 2.06x, 1.95x, 1.84x, respectively. Total speedup of all the benchmarks was 2.16x. As we see in Figure 5, benchmarks for which IKOS took longer to analyze tended to have greater speedup in Pikos $\langle 4\rangle$. Top 25\% benchmarks in terms of the analysis time in IKOS had higher averages than the total benchmarks, with arithmetic, geometric, and harmonic mean of $2.38 \mathrm{x}, 2.29 \mathrm{x}$, and $2.18 \mathrm{x}$, respectively. Table 2 shows the results for benchmarks with the highest speedup and the longest analysis time in IKOS. 


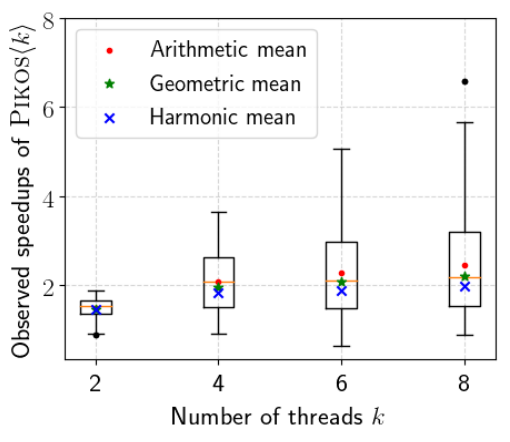

(a) Box plot

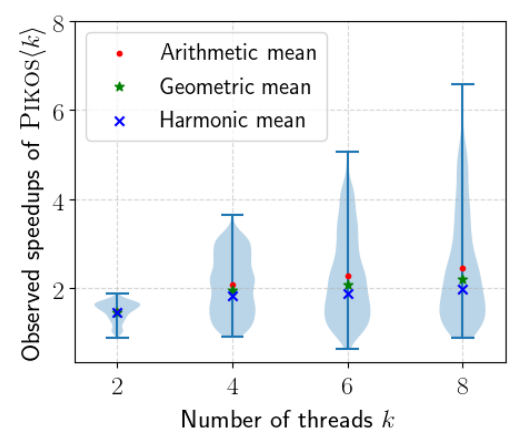

(b) Violin plot

Fig. 7. Box and violin plot for speedup of PIKOs $\langle k\rangle$ with $k \in\{2,4,6,8\}$.

Figure 6 provides details about the distribution of the speedup achieved by Pikos $\langle 4\rangle$. Frequency on $\mathrm{y}$-axis represents the number of benchmarks that have speedups in the bucket on $\mathrm{x}$-axis. A bucket size of 0.25 is used, ranging from 0.75 to 3.75 . Benchmarks are divided into 4 ranges using the analysis time in IKOS, where $0 \%$ represents the benchmark with the shortest analysis time in IKOS and $100 \%$ represents the longest. The longer the analysis time was in IKOS (higher percentile), the more the distribution tended toward a higher speedup for PIKOs $\langle 4\rangle$. The most frequent bucket was $1.25 \mathrm{x}-1.50 \mathrm{x}$ with frequency of 52 for the range $0 \% \sim 25 \%$. For the range $25 \% \sim 50 \%$, it was $1.50 \mathrm{x}-1.75 \mathrm{x}$ with frequency of 45 ; for the range $50 \% \sim 75 \%, 3.00 x-3.25 \mathrm{x}$ with frequency of 38 ; and for the range $75 \% \sim 100 \%$, 3.00x-3.25x with frequency of 50. Overall, Pikos $\langle 4\rangle$ had speedup over 2.00x for 533 benchmarks out of 1017 (52.4\%), and had speedup over 3.00x for 106 out of 1017 (10.4\%).

Benchmarks with high speedup contained code with large switch statements nested inside loops. For example, ratpoison-1.4.9/ratpoison, a tiling window manager, had an event handling loop that dispatches the events using the switch statement with 15 cases. Each switch case called an event handler that contained further branches, leading to more parallelism. Most of the analysis time for this benchmark was spent in this loop. On the other hand, benchmarks with low speedup usually had a dominant single execution path. An example of such a benchmark is xlockmore-5.56/xlock, a program that locks the local X display until a password is entered.

Arithmetic, geometric, and harmonic mean of the speedup exhibited by $\operatorname{Pikos}\langle 4\rangle$ were 2.06x, $1.95 \mathrm{x}, 1.84 \mathrm{x}$, respectively. Maximum speedup exhibited was $3.63 \mathrm{x}$, where $4.00 \mathrm{x}$ is the maximum possible speedup. The performance was generally better for the benchmarks for which IKOS took longer to analyze.

\subsection{RQ2: Scalability of PIkos}

To understand how the performance of PIKos $\langle k\rangle$ scales with the number of threads $k$, we carried out the same measurements as in RQ1 using PIKOs $\langle k\rangle$ with $k \in\{2,4,6,8\}$.

Figure 7 shows the box and violin plots for speedup obtained by PIKоs $\langle k\rangle, k \in\{2,4,6,8\}$. Box plots show the quartiles and the outliers, and violin plots show the estimated distribution of the observed speedups. The box plot [Tukey 1977] on the left summarizes the distribution of the results for each $k$ using lower inner fence $(Q 1-1.5 *(Q 3-Q 1))$, first quartile $(Q 1)$, median, third quartile $(Q 3)$, and upper inner fence $(Q 3+1.5 *(Q 3-Q 1))$. Data beyond the inner fences (outliers) are plotted as individual points. Box plot revealed that while the benchmarks above the median (middle line in the 


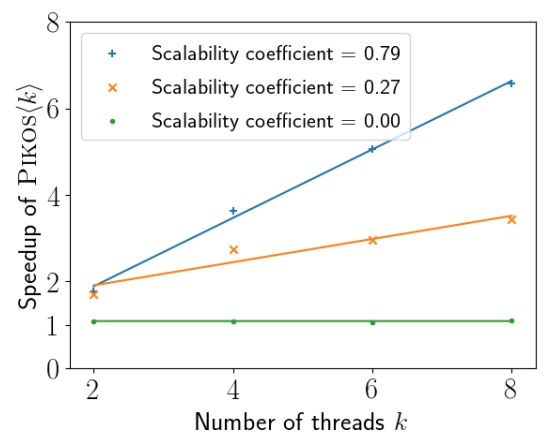

(a) Speedup of PIKOS $\langle k\rangle$ for 3 benchmarks with different scalability coefficients. The lines show the linear regressions of these benchmarks.

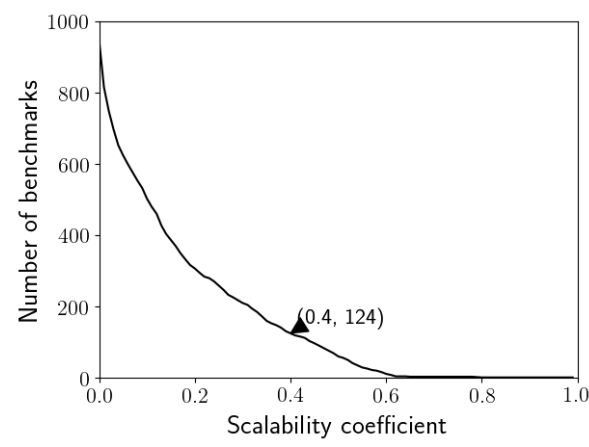

(b) Distribution of scalability coefficients for 1017 benchmarks. $(x, y)$ in the plot means that $y$ number of benchmarks have scalability coefficient at least $x$.

Fig. 8. Scalability coefficient for PІкоs $\langle k\rangle$.

Table 3. Five benchmarks with the highest scalability out of 1017 benchmarks.

\begin{tabular}{lrrrrrr}
\hline & & & \multicolumn{4}{c}{ Speedup of PIKOs $\langle k\rangle$} \\
\cline { 5 - 7 } Benchmark (5 out of 1017. Criteria: Scalability) & Src. & IKOS (s) & $k=4$ & $k=8$ & $k=12$ & $k=16$ \\
\hline audit-2.8.4/aureport & OSS & 684.29 & $3.63 \mathrm{x}$ & $6.57 \mathrm{x}$ & $9.02 \mathrm{x}$ & $10.97 \mathrm{x}$ \\
feh-3.1.3/feh.bc & OSS & 9004.83 & $3.55 \mathrm{x}$ & $6.57 \mathrm{x}$ & $8.33 \mathrm{x}$ & $9.39 \mathrm{x}$ \\
ratpoison-1.4.9/ratpoison & OSS & 1303.73 & $3.36 \mathrm{x}$ & $5.65 \mathrm{x}$ & $5.69 \mathrm{x}$ & $5.85 \mathrm{x}$ \\
ldv-linux-4.2-rc1/32_7a-net-ethernet-intel-igb & SVC & 1206.27 & $3.10 \mathrm{x}$ & $5.44 \mathrm{x}$ & $5.71 \mathrm{x}$ & $6.46 \mathrm{x}$ \\
ldv-linux-4.2-rc1/08_1a-net-wireless-mwifiex & SVC & 10224.21 & $3.12 \mathrm{x}$ & $5.35 \mathrm{x}$ & $6.20 \mathrm{x}$ & $6.64 \mathrm{x}$ \\
\hline
\end{tabular}

box) scaled, speedups for the ones below median saturated. Violin plot [Hintze and Nelson 1998] on the right supplements the box plot by plotting the probability density of the results between minimum and maximum. In the best case, speedup scaled from $1.77 \mathrm{x}$ to $3.63 \mathrm{x}, 5.07 \mathrm{x}$, and $6.57 \mathrm{x}$. For $k \in\{2,4,6,8\}$, the arithmetic means were $1.48 \mathrm{x}, 2.06 \mathrm{x}, 2.26 \mathrm{x}$, and $2.46 \mathrm{x}$, respectively; the geometric means were $1.46 \mathrm{x}, 1.95 \mathrm{x}, 2.07 \mathrm{x}$, and $2.20 \mathrm{x}$, respectively; and the harmonic means were $1.44 \mathrm{x}, 1.84 \mathrm{x}$, $1.88 \mathrm{x}$, and $1.98 \mathrm{x}$, respectively.

To better measure the scalability of PIKOs $\langle k\rangle$ for individual benchmarks, we define a scalability coefficient as the slope of the linear regression of the number of threads and the speedups. The maximum scalability coefficient is 1 , meaning that the speedup increases linearly with the number of threads. If the scalability coefficient is 0 , the speedup is the same regardless of the number of threads used. If it is negative, the speedup goes down with increase in number of threads. The measured scalability coefficients are shown in Figure 8. Figure 8(a) illustrates benchmarks exhibiting different scalability coefficients. For the benchmark with coefficient 0.79 , the speedup of Pikos roughly increases by 4 , from $2 x$ to $6 x$, with 6 more threads. For benchmark with coefficient 0 , the speedup does not increase with more threads. Figure $8(\mathrm{~b})$ shows the distribution of scalability coefficients for all benchmarks. From this plot we can infer, for instance, that 124 benchmarks have at least 0.4 scalability coefficient. For these benchmarks, speedups increased by at least 2 when 5 more threads are given.

Table 3 shows the speedup of Pikos $\langle k\rangle$ for $k \geq 4$ for a selection of five benchmarks that had the highest scalability coefficient in the prior experiment. In particular, we wanted to explore the limits 


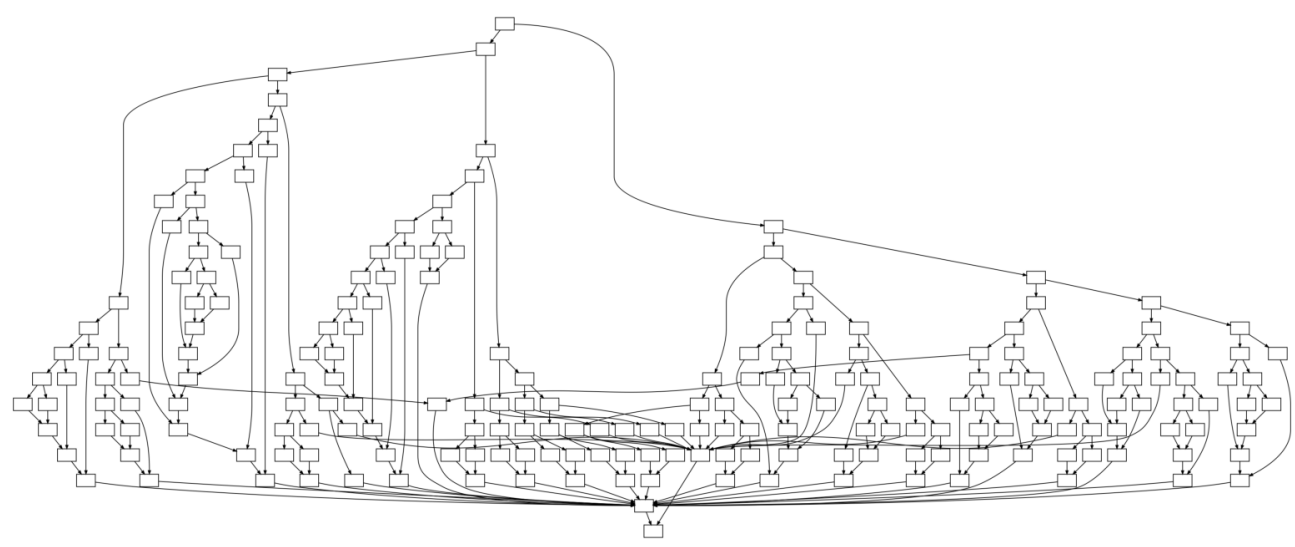

Fig. 9. CFG of function per_event_detailed in aureport for which PIKоs had the maximum scalability. This function calls the appropriate handler based on the event type. This function is called inside a loop.

of scalability of Pikos $\langle k\rangle$ for this smaller selection of benchmarks. With scalability coefficient 0.79 , the speedup of audit-2.8.4/aureport reached 10.97x using 16 threads. This program is a tool that produces summary reports of the audit system logs. Like ratpoison, it has an event-handler loop consisting of a large switch statement as shown in Figure 9.

The scalability of Pıкоs depends on the structure of the analyzed programs. Pikos exhibited high scalability on programs with multiple disjoint paths of similar length. For the program aureport, which had the highest scalability, the speedup of PIKos $\langle k\rangle$ scaled from 1.77x to $3.63 \mathrm{x}, 5.07 \mathrm{x}, 6.57 \mathrm{x}, 9.02 \mathrm{x}$, and $10.97 \mathrm{x}$ with $k=2,4,6,8,12$, and 16 .

\section{RELATED WORK}

Since its publication in 1993, Bourdoncle's algorithm [Bourdoncle 1993] has become the de facto approach to solving equations in abstract interpretation. Many advances have been developed since, but they rely on Bourdoncle's algorithm; in particular, different ways of intertwining widening and narrowing during fixpoint computation with an aim to improve precision [Amato and Scozzari 2013; Amato et al. 2016; Halbwachs and Henry 2012].

C Global Surveyor (CGS) [Venet and Brat 2004] that performed array bounds checking was the first attempt at distributed abstract interpretation. It performed distributed batch processing, and a relational database was used for both storage and communication between processes. Thus, the communication costs were too high, and the analysis did not scale beyond four CPUs.

Monniaux [2005] describes a parallel implementation of the ASTRÉE analyzer [Cousot et al. 2005]. It relies on dispatch points that divide the control flow between long executions, which are found in embedded applications; the tool analyzes these two control paths in parallel. Unlike our approach, this parallelization technique is not applicable to programs with irreducible CFGs. The particular parallelization strategy can also lead to a loss in precision. The experimental evaluation found that the analysis does not scale beyond 4 processors.

Dewey et al. [2015] present a parallel static analysis for JavaScript by dividing the analysis into an embarrassingly parallel reachability computation on a state transition system, and a strategy for selectively merging states during that reachability computation.

Prior work has explored the use of parallelism for specific program analysis. BOLT [Albarghouthi et al. 2012] uses a map-reduce framework to parallelize a top-down analysis for use in verification 
and software model checking. Graspan [Wang et al. 2017] implements a single-machine disk-based graph system to solve graph reachability problems for interprocedural static analysis. Graspan is not a generic abstract interpreter, and solves data-flow analyses in the IFDS framework [Reps et al. 1995]. Su et al. [2014] describe a parallel points-to analysis via CFL-reachability. Garbervetsky et al. [2017] use an actor-model to implement distributed call-graph analysis.

McPeak et al. [2013] parallelize the Coverity Static Analyzer [Bessey et al. 2010] to run on an 8-core machine by mapping each function to its own work unit. Tricorder [Sadowski et al. 2015] is a cloud-based static-analysis platform used at Google. It supports only simple, intraprocedural analyses (such as code linters), and is not designed for distributed whole-program analysis.

Sparse analysis [Oh et al. 2014, 2012] and database-backed analysis [Weiss et al. 2015] are orthogonal approaches that improve the memory cost of static analysis. Newtonian program analysis [Reps 2018; Reps et al. 2017] provides an alternative to Kleene iteration used in this paper.

\section{CONCLUSION}

We presented a generic, parallel, and deterministic algorithm for computing a fixpoint of an equation system for abstract interpretation. The iteration strategy used for fixpoint computation is constructed from a weak partial order (WPO) of the dependency graph of the equation system. We described an axiomatic and constructive characterization of WPOs, as well as an efficient almostlinear time algorithm for constructing a WPO. This new notion of WPO generalizes Bourdoncle's weak topological order (WTO). We presented a linear-time algorithm to construct a WTO from a WPO, which results in an almost-linear algorithm for WTO construction given a directed graph. The previously known algorithm for WTO construction had a worst-case cubic time-complexity. We also showed that the fixpoint computed using the WPO-based parallel fixpoint algorithm is the same as that computed using the WTO-based sequential fixpoint algorithm.

We presented PIKos, our implementation of a WPO-based parallel abstract interpreter. Using a suite of 1017 open-source programs and SV-COMP 2019 benchmarks, we compared the performance of PIKos against the IKOS abstract interpreter. PIKOs $\langle 4\rangle$ achieves an average speedup of 2.06x over IKOS, with a maximum speedup of 3.63x. PIKOS $\langle 4\rangle$ showed greater than $2.00 \mathrm{x}$ speedup for 533 benchmarks (52.4\%) and greater than 3.00x speedup for 106 benchmarks (10.4\%). PIKOs $\langle 4\rangle$ exhibits a larger speedup when analyzing programs that took longer to analyze using IKOS. PIKOs $\langle 4\rangle$ achieved an average speedup of $1.73 \mathrm{x}$ on programs for which IKOS took less than 16 seconds, while PIKOs $\langle 4\rangle$ achieved an average speedup of $2.38 \mathrm{x}$ on programs for which IKOS took greater than 508 seconds. The scalability of Pikos depends on the structure of the program being analyzed with Pikos $\langle 16\rangle$ exhibiting a maximum speedup of $10.97 \mathrm{x}$.

\section{ACKNOWLEDGMENTS}

The authors would like to thank Maxime Arthaud for help with IKOS. This material is based upon work supported by a Facebook Testing and Verification research award, and AWS Cloud Credits for Research.

\section{REFERENCES}

Aws Albarghouthi, Rahul Kumar, Aditya V. Nori, and Sriram K. Rajamani. 2012. Parallelizing top-down interprocedural analyses. In ACM SIGPLAN Conference on Programming Language Design and Implementation, PLDI '12, Beijing, China fune 11 - 16, 2012. 217-228. https://doi.org/10.1145/2254064.2254091

Gianluca Amato and Francesca Scozzari. 2013. Localizing Widening and Narrowing. In Static Analysis - 20th International Symposium, SAS 2013, Seattle, WA, USA, fune 20-22, 2013. Proceedings. 25-42. https://doi.org/10.1007/978-3-642-38856-9_4

Gianluca Amato, Francesca Scozzari, Helmut Seidl, Kalmer Apinis, and Vesal Vojdani. 2016. Efficiently intertwining widening and narrowing. Sci. Comput. Program. 120 (2016), 1-24. https://doi.org/10.1016/j.scico.2015.12.005

Proc. ACM Program. Lang., Vol. 4, No. POPL, Article 14. Publication date: January 2020. 
Gogul Balakrishnan, Malay K. Ganai, Aarti Gupta, Franjo Ivancic, Vineet Kahlon, Weihong Li, Naoto Maeda, Nadia Papakonstantinou, Sriram Sankaranarayanan, Nishant Sinha, and Chao Wang. 2010. Scalable and precise program analysis at NEC. In Proceedings of 10th International Conference on Formal Methods in Computer-Aided Design, FMCAD 2010, Lugano, Switzerland, October 20-23. 273-274. http://ieeexplore.ieee.org/document/5770960/

Thomas Ball, Byron Cook, Vladimir Levin, and Sriram K. Rajamani. 2004. SLAM and Static Driver Verifier: Technology Transfer of Formal Methods inside Microsoft. In Integrated Formal Methods, 4th International Conference, IFM 2004, Canterbury, UK, April 4-7, 2004, Proceedings. 1-20. https://doi.org/10.1007/978-3-540-24756-2_1

Al Bessey, Ken Block, Benjamin Chelf, Andy Chou, Bryan Fulton, Seth Hallem, Charles-Henri Gros, Asya Kamsky, Scott McPeak, and Dawson R. Engler. 2010. A few billion lines of code later: using static analysis to find bugs in the real world. Commun. ACM 53, 2 (2010), 66-75. https://doi.org/10.1145/1646353.1646374

Dirk Beyer. 2019. Automatic Verification of C and Java Programs: SV-COMP 2019. In Tools and Algorithms for the Construction and Analysis of Systems - 25 Years of TACAS: TOOLympics, Held as Part of ETAPS 2019, Prague, Czech Republic, April 6-11, 2019, Proceedings, Part III. 133-155. https://doi.org/10.1007/978-3-030-17502-3_9

Dirk Beyer, Stefan Löwe, and Philipp Wendler. 2019. Reliable benchmarking: requirements and solutions. STTT 21, 1 (2019), 1-29. https://doi.org/10.1007/s10009-017-0469-y

François Bourdoncle. 1993. Efficient chaotic iteration strategies with widenings. In Formal Methods in Programming and Their Applications, International Conference, Akademgorodok, Novosibirsk, Russia, June 28 - July 2, 1993, Proceedings. 128-141. https://doi.org/10.1007/BFb0039704

Guillaume Brat, Jorge A. Navas, Nija Shi, and Arnaud Venet. 2014. IKOS: A Framework for Static Analysis Based on Abstract Interpretation. In Software Engineering and Formal Methods - 12th International Conference, SEFM 2014, Grenoble, France, September 1-5, 2014. Proceedings. 271-277. https://doi.org/10.1007/978-3-319-10431-7_20

Guillaume Brat and Arnaud Venet. 2005. Precise and Scalable Static Program Analysis of NASA Flight Software. In 2005 IEEE Aerospace Conference. 1-10. https://doi.org/10.1109/AERO.2005.1559604

Thomas H. Cormen, Charles E. Leiserson, Ronald L. Rivest, and Clifford Stein. 2009. Introduction to Algorithms, 3rd Edition. MIT Press. http://mitpress.mit.edu/books/introduction-algorithms

Patrick Cousot. 1977. Asynchronous iterative methods for solving a fixed point system of monotone equations in a complete lattice. Res. rep. R.R. 88. Laboratoire IMAG, Université scientifique et médicale de Grenoble, Grenoble, France. 15 p.

Patrick Cousot. 2015. Abstracting Induction by Extrapolation and Interpolation. In Verification, Model Checking, and Abstract Interpretation - 16th International Conference, VMCAI 2015, Mumbai, India, January 12-14, 2015. Proceedings. 19-42. https://doi.org/10.1007/978-3-662-46081-8_2

Patrick Cousot and Radhia Cousot. 1976. Static determination of dynamic properties of programs. In Proceedings of the Second International Symposium on Programming. Dunod, Paris, France, 106-130.

Patrick Cousot and Radhia Cousot. 1977. Abstract Interpretation: A Unified Lattice Model for Static Analysis of Programs by Construction or Approximation of Fixpoints. In Conference Record of the Fourth ACM Symposium on Principles of Programming Languages, Los Angeles, California, USA, January 1977. 238-252. https://doi.org/10.1145/512950.512973

Patrick Cousot, Radhia Cousot, Jérôme Feret, Laurent Mauborgne, Antoine Miné, David Monniaux, and Xavier Rival. 2005. The ASTREÉ Analyzer. In Programming Languages and Systems, 14th European Symposium on Programming,ESOP 2005, Held as Part of the Joint European Conferences on Theory and Practice of Software, ETAPS 2005, Edinburgh, UK, April 4-8, 2005, Proceedings. 21-30. https://doi.org/10.1007/978-3-540-31987-0_3

Patrick Cousot, Roberto Giacobazzi, and Francesco Ranzato. 2019. A A $^{2}$ : abstract ${ }^{2}$ interpretation. PACMPL 3, POPL (2019), 42:1-42:31. https://doi.org/10.1145/3290355

Patrick Cousot and Nicolas Halbwachs. 1978. Automatic Discovery of Linear Restraints Among Variables of a Program. In Conference Record of the Fifth Annual ACM Symposium on Principles of Programming Languages, Tucson, Arizona, USA, January 1978. 84-96. https://doi.org/10.1145/512760.512770

David Delmas and Jean Souyris. 2007. Astrée: From Research to Industry. In Static Analysis, 14th International Symposium, SAS 2007, Kongens Lyngby, Denmark, August 22-24, 2007, Proceedings. 437-451. https://doi.org/10.1007/978-3-540-74061-2_27

Kyle Dewey, Vineeth Kashyap, and Ben Hardekopf. 2015. A parallel abstract interpreter for JavaScript. In Proceedings of the 13th Annual IEEE/ACM International Symposium on Code Generation and Optimization, CGO 2015, San Francisco, CA, USA, February 07 - 11, 2015. 34-45. https://doi.org/10.1109/CGO.2015.7054185

Graeme Gange, Jorge A. Navas, Peter Schachte, Harald Søndergaard, and Peter J. Stuckey. 2016. An Abstract Domain of Uninterpreted Functions. In Verification, Model Checking, and Abstract Interpretation - 17th International Conference, VMCAI 2016, St. Petersburg, FL, USA, January 17-19, 2016. Proceedings. 85-103. https://doi.org/10.1007/978-3-662-49122-5_4

Diego Garbervetsky, Edgardo Zoppi, and Benjamin Livshits. 2017. Toward full elasticity in distributed static analysis: the case of callgraph analysis. In Proceedings of the 2017 11th foint Meeting on Foundations of Software Engineering, ESEC/FSE 2017, Paderborn, Germany, September 4-8, 2017. 442-453. https://doi.org/10.1145/3106237.3106261

Michael R. Garey and David S. Johnson. 1979. Computers and Intractability: A Guide to the Theory of NP-Completeness. W. H. Freeman 
Roberto Giacobazzi and Isabella Mastroeni. 2004. Abstract non-interference: parameterizing non-interference by abstract interpretation. In Proceedings of the 31st ACM SIGPLAN-SIGACT Symposium on Principles of Programming Languages, POPL 2004, Venice, Italy, January 14-16, 2004. 186-197. https://doi.org/10.1145/964001.964017

Denis Gopan and Thomas W. Reps. 2006. Lookahead Widening. In Computer Aided Verification, 18th International Conference, CAV 2006, Seattle, WA, USA, August 17-20, 2006, Proceedings. 452-466. https://doi.org/10.1007/11817963_41

Philippe Granger. 1989. Static analysis of arithmetical congruences. International fournal of Computer Mathematics 30, 3-4 (1989), 165-190. https://doi.org/10.1080/00207168908803778

Nicolas Halbwachs and Julien Henry. 2012. When the Decreasing Sequence Fails. In Static Analysis - 19th International Symposium, SAS 2012, Deauville, France, September 11-13, 2012. Proceedings. 198-213. https://doi.org/10.1007/978-3-64233125-1_15

Paul Havlak. 1997. Nesting of Reducible and Irreducible Loops. ACM Trans. Program. Lang. Syst. 19, 4 (1997), $557-567$. https://doi.org/10.1145/262004.262005

Matthew S. Hecht and Jeffrey D. Ullman. 1972. Flow Graph Reducibility. SIAM J. Comput. 1, 2 (1972), 188-202. https: //doi.org/10.1137/0201014

Jerry L. Hintze and Ray D. Nelson. 1998. Violin Plots: A Box Plot-Density Trace Synergism. The American Statistician 52, 2 (1998), 181-184. https://doi.org/10.1080/00031305.1998.10480559

Raoul Praful Jetley, Paul L. Jones, and Paul Anderson. 2008. Static Analysis of Medical Device Software Using CodeSonar. In Proceedings of the 2008 Workshop on Static Analysis (SAW'08). ACM, New York, NY, USA, 22-29. https://doi.org/10.1145/ 1394504.1394507

Richard M. Karp. 1972. Reducibility Among Combinatorial Problems. In Proceedings of a symposium on the Complexity of Computer Computations, held March 20-22, 1972, at the IBM Thomas f. Watson Research Center, Yorktown Heights, New York, USA. 85-103. https://doi.org/10.1007/978-1-4684-2001-2_9

Sol Kim, Kihong Heo, Hakjoo Oh, and Kwangkeun Yi. 2016. Widening with thresholds via binary search. Softw., Pract. Exper. 46, 10 (2016), 1317-1328. https://doi.org/10.1002/spe.2381

Scott McPeak, Charles-Henri Gros, and Murali Krishna Ramanathan. 2013. Scalable and incremental software bug detection. In foint Meeting of the European Software Engineering Conference and the ACM SIGSOFT Symposium on the Foundations of Software Engineering, ESEC/FSE'13, Saint Petersburg, Russian Federation, August 18-26, 2013. 554-564. https://doi.org/10. $1145 / 2491411.2501854$

Antoine Miné. 2001. A New Numerical Abstract Domain Based on Difference-Bound Matrices. In Programs as Data Objects, Second Symposium, PADO 2001, Aarhus, Denmark, May 21-23, 2001, Proceedings. 155-172. https://doi.org/10.1007/3-54044978-7_10

Antoine Miné. 2004. Relational Abstract Domains for the Detection of Floating-Point Run-Time Errors. In Programming Languages and Systems, 13th European Symposium on Programming, ESOP 2004, Held as Part of the foint European Conferences on Theory and Practice of Software, ETAPS 2004, Barcelona, Spain, March 29 - April 2, 2004, Proceedings. 3-17. https://doi.org/10.1007/978-3-540-24725-8_2

Antoine Miné. 2006. The octagon abstract domain. Higher-Order and Symbolic Computation 19, 1 (2006), 31-100. https: //doi.org/10.1007/s10990-006-8609-1

David Monniaux. 2005. The Parallel Implementation of the Astrée Static Analyzer. In Programming Languages and Systems, Third Asian Symposium, APLAS 2005, Tsukuba, Japan, November 2-5, 2005, Proceedings. 86-96. https://doi.org/10.1007/ $11575467 \_7$

Hakjoo Oh, Kihong Heo, Wonchan Lee, Woosuk Lee, Daejun Park, Jeehoon Kang, and Kwangkeun Yi. 2014. Global Sparse Analysis Framework. ACM Trans. Program. Lang. Syst. 36, 3 (2014), 8:1-8:44. https://doi.org/10.1145/2590811

Hakjoo Oh, Kihong Heo, Wonchan Lee, Woosuk Lee, and Kwangkeun Yi. 2012. Design and implementation of sparse global analyses for C-like languages. In ACM SIGPLAN Conference on Programming Language Design and Implementation, PLDI '12, Beijing, China - June 11 - 16, 2012. 229-238. https://doi.org/10.1145/2254064.2254092

Mendes Oulamara and Arnaud J. Venet. 2015. Abstract Interpretation with Higher-Dimensional Ellipsoids and Conic Extrapolation. In Computer Aided Verification - 27th International Conference, CAV 2015, San Francisco, CA, USA, fuly 18-24, 2015, Proceedings, Part I. 415-430. https://doi.org/10.1007/978-3-319-21690-4_24

Ganesan Ramalingam. 1999. Identifying Loops in Almost Linear Time. ACM Trans. Program. Lang. Syst. 21, 2 (1999), $175-188$. https://doi.org/10.1145/316686.316687

Ganesan Ramalingam. 2002. On loops, dominators, and dominance frontiers. ACM Trans. Program. Lang. Syst. 24, 5 (2002), 455-490. https://doi.org/10.1145/570886.570887

James Reinders. 2007. Intel threading building blocks - outfitting C++ for multi-core processor parallelism. O'Reilly. http: //www.oreilly.com/catalog/9780596514808/index.html

Thomas W. Reps. 2018. Program Analyses Using Newton's Method (Invited Paper). In Networked Systems - 6th International Conference, NETYS 2018, Essaouira, Morocco, May 9-11, 2018, Revised Selected Papers. 3-16. https://doi.org/10.1007/978-3030-05529-5_1

Proc. ACM Program. Lang., Vol. 4, No. POPL, Article 14. Publication date: January 2020. 
Thomas W. Reps, Susan Horwitz, and Mooly Sagiv. 1995. Precise Interprocedural Dataflow Analysis via Graph Reachability. In Conference Record of POPL'95: 22nd ACM SIGPLAN-SIGACT Symposium on Principles of Programming Languages, San Francisco, California, USA, January 23-25, 1995. 49-61. https://doi.org/10.1145/199448.199462

Thomas W. Reps, Emma Turetsky, and Prathmesh Prabhu. 2017. Newtonian Program Analysis via Tensor Product. ACM Trans. Program. Lang. Syst. 39, 2 (2017), 9:1-9:72. https://doi.org/10.1145/3024084

Noam Rinetzky, Jörg Bauer, Thomas W. Reps, Mooly Sagiv, and Reinhard Wilhelm. 2005. A semantics for procedure local heaps and its abstractions. In Proceedings of the 32nd ACM SIGPLAN-SIGACT Symposium on Principles of Programming Languages, POPL 2005, Long Beach, California, USA, fanuary 12-14, 2005. 296-309. https://doi.org/10.1145/1040305.1040330

Caitlin Sadowski, Jeffrey van Gogh, Ciera Jaspan, Emma Söderberg, and Collin Winter. 2015. Tricorder: Building a Program Analysis Ecosystem. In 37th IEEE/ACM International Conference on Software Engineering, ICSE 2015, Florence, Italy, May 16-24, 2015, Volume 1. 598-608. https://doi.org/10.1109/ICSE.2015.76

Gagandeep Singh, Markus Püschel, and Martin T. Vechev. 2017. Fast polyhedra abstract domain. In Proceedings of the 44th ACM SIGPLAN Symposium on Principles of Programming Languages, POPL 2017, Paris, France, January 18-20, 2017. 46-59. http://dl.acm.org/citation.cfm?id=3009885

Gagandeep Singh, Markus Püschel, and Martin T. Vechev. 2018a. Fast Numerical Program Analysis with Reinforcement Learning. In Computer Aided Verification - 30th International Conference, CAV 2018, Held as Part of the Federated Logic Conference, FloC 2018, Oxford, UK, fuly 14-17, 2018, Proceedings, Part I. 211-229. https://doi.org/10.1007/978-3-319-961453_12

Gagandeep Singh, Markus Püschel, and Martin T. Vechev. 2018b. A practical construction for decomposing numerical abstract domains. PACMPL 2, POPL (2018), 55:1-55:28. https://doi.org/10.1145/3158143

Yu Su, Ding Ye, and Jingling Xue. 2014. Parallel Pointer Analysis with CFL-Reachability. In 43rd International Conference on Parallel Processing, ICPP 2014, Minneapolis, MN, USA, September 9-12, 2014. 451-460. https://doi.org/10.1109/ICPP.2014.54

Edward Szpilrajn. 1930. Sur l'extension de l'ordre partiel. Fundamenta Mathematicae 16, 1 (1930), 386-389. http://eudml. org/doc/212499

Robert Endre Tarjan. 1973. Testing Flow Graph Reducibility. In Proceedings of the 5th Annual ACM Symposium on Theory of Computing, April 30 - May 2, 1973, Austin, Texas, USA. 96-107. https://doi.org/10.1145/800125.804040

Robert Endre Tarjan. 1979. Applications of Path Compression on Balanced Trees. J. ACM 26, 4 (1979), 690-715. https: //doi.org/10.1145/322154.322161

John W. Tukey. 1977. Exploratory data analysis (Addison-Wesley series in behavioral science : quantitative methods). AddisonWesley. http://www.worldcat.org/oclc/03058187

Arnaud Venet. 2012. The Gauge Domain: Scalable Analysis of Linear Inequality Invariants. In Computer Aided Verification 24th International Conference, CAV 2012, Berkeley, CA, USA, July 7-13, 2012 Proceedings. 139-154. https://doi.org/10.1007/ 978-3-642-31424-7_15

Arnaud Venet and Guillaume P. Brat. 2004. Precise and efficient static array bound checking for large embedded C programs. In Proceedings of the ACM SIGPLAN 2004 Conference on Programming Language Design and Implementation 2004, Washington, DC, USA, June 9-11, 2004. 231-242. https://doi.org/10.1145/996841.996869

Kai Wang, Aftab Hussain, Zhiqiang Zuo, Guoqing (Harry) Xu, and Ardalan Amiri Sani. 2017. Graspan: A Single-machine Disk-based Graph System for Interprocedural Static Analyses of Large-scale Systems Code. In Proceedings of the TwentySecond International Conference on Architectural Support for Programming Languages and Operating Systems, ASPLOS 2017, Xi'an, China, April 8-12, 2017. 389-404. https://doi.org/10.1145/3037697.3037744

Cathrin Weiss, Cindy Rubio-González, and Ben Liblit. 2015. Database-Backed Program Analysis for Scalable Error Propagation. In 37th IEEE/ACM International Conference on Software Engineering, ICSE 2015, Florence, Italy, May 16-24, 2015, Volume 1. 586-597. https://doi.org/10.1109/ICSE.2015.75

Reinhard Wilhelm, Shmuel Sagiv, and Thomas W. Reps. 2000. Shape Analysis. In Compiler Construction, 9th International Conference, CC 2000, Held as Part of the European foint Conferences on the Theory and Practice of Software, ETAPS 2000, Berlin, Germany, March 25 - April 2, 2000, Proceedings. 1-17. https://doi.org/10.1007/3-540-46423-9_1 